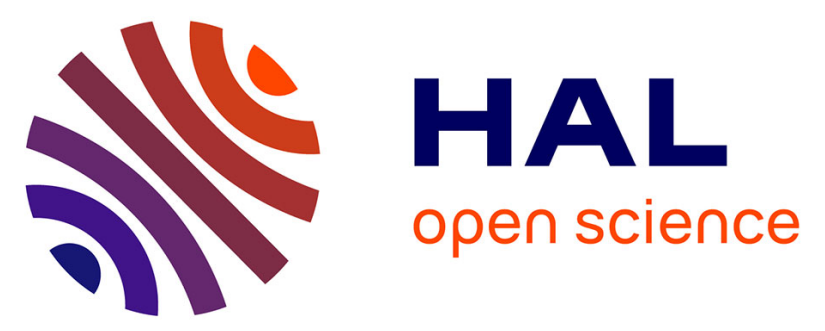

\title{
Chikungunya virus envelope protein E2 provides a vector for targeted antigen delivery to human dermal CD14 + dendritic cells
}

Adrien Brulefert, Melanie Kraemer, Marie Cumin, Amandine Selle, Astrid Hoste, Hans-Henrik Gad, Julia Rühl, Jean-Baptiste Madinier, Olivier Chaloin, Christian Münz, et al.

\section{To cite this version:}

Adrien Brulefert, Melanie Kraemer, Marie Cumin, Amandine Selle, Astrid Hoste, et al.. Chikungunya virus envelope protein E2 provides a vector for targeted antigen delivery to human dermal CD14 + dendritic cells. Journal of Investigative Dermatology, 2021, 10.1016/j.jid.2021.04.027 . hal-03259616

\section{HAL Id: hal-03259616 https://hal.science/hal-03259616}

Submitted on 14 Jun 2021

HAL is a multi-disciplinary open access archive for the deposit and dissemination of scientific research documents, whether they are published or not. The documents may come from teaching and research institutions in France or abroad, or from public or private research centers.
L'archive ouverte pluridisciplinaire HAL, est destinée au dépôt et à la diffusion de documents scientifiques de niveau recherche, publiés ou non, émanant des établissements d'enseignement et de recherche français ou étrangers, des laboratoires publics ou privés. 
Chikungunya virus envelope protein E2 provides a vector for targeted antigen delivery to human dermal CD14+ dendritic cells.

Adrien Brulefert ${ }^{1}$, Melanie Kraemer ${ }^{1}$, Marie Cumin ${ }^{1}$, Amandine Selle ${ }^{1}$, Astrid Hoste ${ }^{1}$, Hans-Henrik Gad ${ }^{2}$, Julia Rühl${ }^{3}$, Jean-Baptiste Madinier ${ }^{1}$, Olivier Chaloin ${ }^{1}$, Christian Münz ${ }^{3}$, Philippe Desprès ${ }^{2,4}$, Christopher George Mueller $^{1}$, Vincent Flacher ${ }^{1 *}$

1. Laboratory CNRS I²T/UPR3572 Immunology, Immunopathology and Therapeutic Chemistry, Strasbourg Drug Discovery and Development Institute (IMS), Institut de Biologie Moléculaire et Cellulaire, Strasbourg, France

2. Unité Interactions Moléculaires Flavivirus-Hôtes, Institut Pasteur, Paris, France.

3. Viral Immunobiology, Institute of Experimental Immunology, University of Zürich, Zürich, Switzerland.

4. Present address: Université de La Réunion, INSERM U1187, CNRS UMR 9192, IRD UMR 249, Unité Mixte Processus Infectieux en Milieu Insulaire Tropical, Plateforme Technologique CYROI, 97491 Sainte-Clotilde, La Réunion, France.

*Correspondence: v.flacher@ibmc-cnrs.unistra.fr

1006 words 


\section{TO THE EDITOR}

Therapeutic modulation of human cutaneous antigen-presenting cells (APCS) that control the fate of T cells represents an interesting option for immunotherapy. As they capture glycosylated proteins via C-type lectins, lectin-targeting antibodies have been used to specifically convey antigens to APCs (Romani et al., 2012;Stoitzner et al., 2014;Kastenmuller et al., 2014). Beyond potential therapeutic applications, APCtargeting strategies can be harnessed to acquire fundamental knowledge about their function (Flacher et al., 2014). In human skin, epidermal Langerhans cells (LCs), CD1c dermal type-2 conventional dendritic cells (cDC2) and CD141+ dermal CDC1 (Nestle et al., 2009;Schlitzer et al., 2015) can migrate to lymph nodes and stimulate T cells. Dermal APCs (dAPCs) include two CD14+ subsets that express the lectins Mannose Receptor (MR)/CD206 and DC-specific intracellular adhesion molecules-3 grabbing non-integrin (DC-SIGN)/CD209 (Klechevsky et al., 2008;McGovern et al., 2014). During skin explant culture, CD14+ dermal DCs (dDCs) migrate into the supernatant, whereas sessile dermal macrophages require enzymatic digestion to be isolated. Still, CD14 ${ }^{+} \mathrm{dDCs}$ and dermal macrophages display strong similarities (McGovern et al., 2014). They are both considered permanent dermal residents that exert local functions in pathogen capture (e.g. arthropod-borne viruses) (Kwan et al., 2008;Schaeffer et al., 2015) and may interact with skin-resident memory T cells (TRM) (Clark, 2015). Subset differences might reflect their origin, i.e. monocyte-derived dDCs vs. embryonically seeded resident macrophages (Ginhoux and Guilliams, 2016;McGovern et al., 2014). Because tools to specifically target $\mathrm{CD} 14^{+} \mathrm{dAPCs}$ remain scarce (Duinkerken et al., 2019), we propose here a previously unreported approach based on recombinant arboviral envelope proteins.

Chikungunya virus (CHIKV) of the flavivirus genus of Togaviridae family is introduced into the dermis during blood meals of mosquitoes. CHIKV infection triggers high fever, skin rash and persistent arthralgia. Our finding that $C D 14^{+}$dAPCs are preferentially infected by Dengue virus (Kwan et al., 2008;Schaeffer et al., 2015) prompted the idea that they may represent an immediate CHIKV target. CD14 ${ }^{+} \mathrm{dAPCs}$ isolated from human dermis, monocyte-derived DCs (MDDCs) and dermal-like macrophages (MDdMs) were exposed to CHIKV06.049 strain $(\mathrm{MOI}=2.5)$ and compared with HeLa cells, in which vigorous viral replication is expected (Sourisseau et al., 2007). CD14 ${ }^{+}$dAPCs and their monocyte-derived equivalent MDdMs were permissive CHIKV target cells, as well as MDDCs, which resemble the inflammatory DCs recruited to the site of infection (Figure 1a).

We hypothesized that APCs express endocytic receptors of viral envelope proteins. CHIK-sE2, a soluble form of CHIKV envelope glycoprotein E2, was fused to the SNAP proprietary enzyme and produced in insect cells to retain specific $\mathrm{N}$-glycosylations that may be recognized by lectins (Figure 1b). In surface plasmon resonance assays, CHIK-sE2 displayed high affinity for MR and DC-SIGN (Figure S1a/b), with dissociation constants of $3.9 \mathrm{nM}$ and $51.1 \mathrm{nM}$, respectively (Figure S1c). CHIK-sE2 was then covalently conjugated via its enzymatic SNAP moiety to AlexaFluor488 fluorochrome (Figure 1c). Since MDdMs and MDDCs, but not 
monocytes, express high levels of lectins (Kwan et al., 2008), we assessed their binding of the recombinant viral glycoprotein. CHIK-sE2-A488 only labelled MDdMs and MDDCs, and an anti-CHIK-sE2 antibody diminished binding to MDDCs (Figure 1d/e). Moreover, fluorescence microscopy revealed intracellular vesicles containing CHIK-sE2-A488 within MDdMs and MDDCs, but not in monocytes (Figure 1f). Incubation with CHIK-sE2 had no influence on MDDC maturation, estimated by CD86 expression (Figure S2).

To evaluate whether CHIK-sE2 can target CD14+ $\mathrm{dAPCS}$ within the skin, we compared its capture by different primary cutaneous APCs and non-hematopoietic cells. Epidermal cell suspensions, fibroblasts or purified CD14 ${ }^{+}$dAPCs and CD1 $c^{+}$dDCs were incubated with CHIK-sE2-A488 and binding efficacy was measured by flow cytometry. CD14+ dAPCs displayed clear binding of CHIK-sE2-A488, as opposed to keratinocytes, LCs, fibroblasts and CD1c dDCs (Figure 1g). Anti-CHIK-sE2 mAb inhibited the interaction with CD14+ dAPCs. Quantitative analyses on skin samples from different individuals confirmed that only CD14+ $d A P C s$ could efficiently capture CHIK-sE2-A488 (Figure 1h). CHIK-sE2-A488 was detected as condensed spots in the cytoplasm of CD14+ dAPCs, reflecting endocytic uptake (Figure 1i).

We next tested whether CHIK-sE2 could be used to convey antigens to CD14 ${ }^{+} \mathrm{dDCs}$, which stimulate T cells more potently than macrophages (McGovern et al., 2014). To assess binding to CD14+ $\mathrm{dDCs}$ in comparison with the other skin DC subsets, we cultured skin explants for 3 days and collected LCs, CD14- dDCs and CD14+ dDCs from the supernatant (Figure 2a). When the cell suspension was exposed to CHIK-sE2-A488, only CD14+ dDCs significantly captured (Figure $\mathbf{2 b} / \mathbf{c}$ ) and internalized (Figure $\mathbf{2 d}$, Video $\mathbf{5 3}$ ) the fluorescent protein. Mannan potently decreased CHIK-sE2-A488 binding, and inhibition assays using anti-lectin mAb suggested that MR is a more efficient CHIK-sE2 receptor than DC-SIGN (Figure 2e).

To address antigen presentation, we used a $\mathrm{CD}^{+}$T-cell clone with defined specificity for HLA-DR1restricted antigenic peptide of the Epstein-Barr virus Nuclear Antigen-1 (EBNA1) (Rühl et al., 2019). Total migratory APCs or CD14+ dDCs from HLA-DR1 ${ }^{+}$skin explants (5 donors out of 34 ) were loaded with CHIK-sE2 conjugated to EBNA1 peptide (Figure 2f). APCs were then cocultured with CFSE-labelled, EBNA1-specific CD4 ${ }^{+}$ T cells. After 5 days, proliferation and intracellular IFN- $\gamma$ production were quantified by flow cytometry. CHIKsE2-EBNA1-exposed APCs from HLA-DR1 donors strongly enhanced proliferation (Figure $\mathbf{2 g}$ ) and triggered IFN$\checkmark$ production (Figure $\mathbf{2} \mathbf{h}$ ) of EBNA1-specific $\mathrm{T}$ cells. However, $\mathrm{T}$ cells did not proliferate when cultured with mismatched, non-HLA-DR1 APCs (Figure 2i). Targeting purified $\mathrm{CD}_{14}^{+} \mathrm{dDCs}$ with CHIK-sE2-EBNA1 was sufficient to promote EBNA1-specific T-cell proliferation. Yet, IFN- $\gamma$ production was not significantly enhanced, possibly because CD14 $4^{+} \mathrm{dDC}$ produce anti-inflammatory IL-10 and display low CD86 expression (Klechevsky et al., 2008).

Cutaneous TRM are derived from effector T cells that colonize the skin to mediate adaptive immune responses (Clark, 2015). CD14 $4^{+}$dAPCs might be critically involved during secondary exposure to cognate antigen because they also permanently reside in the skin and can reactivate memory T cells (McGovern et al., 
2014). CD14+ dAPCs targeting by CHIK-sE2 could be particularly useful to investigate and influence these interactions for therapeutic purposes. Moreover, our work suggests that arboviruses may represent a natural reservoir of molecules targeting different skin APCs. They may be derived into a specific delivery system for antigens but also adjuvants, silencing RNA or toxins, thereby harnessing the properties of skin APCs for immunotherapy or vaccination.

\section{ORCID ID}

Adrien Brulefert: https://orcid.org/0000-0002-3307-4298

Melanie Kraemer : https://orcid.org/0000-0002-0208-7029

Marie Cumin : https://orcid.org/0000-0002-4776-8231

Amandine Selle: https://orcid.org/0000-0002-9057-8580

Astrid Hoste: https://orcid.org/0000-0002-3531-1285

Hans-Henrik Gad: https://orcid.org/0000-0001-8449-1115

Julia Rühl: https://orcid.org/0000-0001-8385-4367

Jean-Baptiste Madinier: https://orcid.org/0000-0001-9503-2619

Olivier Chaloin: https://orcid.org/0000-0003-3045-4452

Christian Münz: https://orcid.org/0000-0001-6419-1940

Philippe Desprès: https://orcid.org/0000-0001-8926-4050

Christopher George Mueller: https://orcid.org/0000-0002-4119-2729

Vincent Flacher: https://orcid.org/0000-0002-8648-2615

\section{Data availability}

No datasets were generated or analyzed during the current study.

\section{Conflict of Interest Statement}

The authors declare no conflict of interest.

\section{Acknowledgements}

PD, CGM and VF designed the research. JR and CM provided EBNA-1-specific $T$ cell lines and methods for antigen-specific assays. JBM and OC produced antigenic peptides. OC performed surface plasmon resonance assays. $A B, M K, M C, A S, A H, H H G, C G M$ and VF performed experiments. $A B, M K, C G M$ and $V F$ analyzed the data. AB, PD, CGM and VF wrote the manuscript. The authors wish to thank Dr. Teunis Geijtenbeek (University of Amsterdam, NL) for anti-DC-SIGN mAb clone AZN-D1, as well as Drs. Sylvie Tourne and Henri De La Salle (Etablissement Français du Sang, Strasbourg, France) for their advice on HLA typing assays. This work was 
supported by the European Union Marie-Slodowska-Curie Career Integration Grant of Dr. V. Flacher (PCIG12GA-2012-334011 - DERMACRO) and by the Agence Nationale pour la Recherche (Program "Investissements d'Avenir" ANR-10-LABX-0034 MEDALIS and ANR-11-EQPX-022; ANR ERAPerMed ANR-18-PERM-0001 BATMAN).

\section{CRediT statement}

Conceptualization: PD, CGM, VF; Data Curation: AB, CGM, VF; Formal Analysis: AB, CGM, VF; Funding Acquisition: CGM, VF; Investigation: $A B, M K, M C, A S, A H, H H G, C G M, V F$; Methodology: OC, CM, PD, CGM, VF; Project administration: CGM, VF; Resources: JBM, OC, PD, JR, CM, PD; Supervision: PD, CGM, VF; Validation: $A B, M K, M C, A S, A H, H H G, J R, J B M, O C, C M, P D, C G M, V F$; Visualization: AB, OC, CGM, VF; Writing - Original Draft Preparation: $A B, P D, C G M, V F$; Writing - Review and Editing: $A B, P D, C G M, V F$. 


\section{REFERENCES}

Clark RA. Resident memory T cells in human health and disease. Sci Transl Med 2015;7:269rv1.

Duinkerken S, Horrevorts SK, Kalay H, Ambrosini M, Rutte L, De Gruijl TD, et al. Glyco-Dendrimers as Intradermal Anti-Tumor Vaccine Targeting Multiple Skin DC Subsets. Theranostics 2019;9:5797-5809.

Flacher V, Tripp CH, Mairhofer DG, Steinman RM, Stoitzner P, Idoyaga J, et al. Murine Langerin+ dermal dendritic cells prime CD8+ T cells while Langerhans cells induce cross-tolerance. EMBO Mol Med 2014;6:11911204.

Ginhoux F, Guilliams M. Tissue-Resident Macrophage Ontogeny and Homeostasis. Immunity 2016;44:439-449. Kastenmuller W, Kastenmuller K, Kurts C, Seder RA. Dendritic cell-targeted vaccines--hope or hype? Nat Rev Immunol 2014;14:705-711.

Klechevsky E, Morita R, Liu M, Cao Y, Coquery S, Thompson-Snipes L, et al. Functional specializations of human epidermal Langerhans cells and CD14+ dermal dendritic cells. Immunity 2008;29:497-510.

Kwan WH, Navarro-Sanchez E, Dumortier H, Decossas M, Vachon H, dos Santos FB, et al. Dermal-type macrophages expressing CD209/DC-SIGN show inherent resistance to dengue virus growth. PLoS Negl Trop Dis 2008;2:e311.

McGovern N, Schlitzer A, Gunawan M, Jardine L, Shin A, Poyner E, et al. Human dermal CD14(+) cells are a transient population of monocyte-derived macrophages. Immunity 2014;41:465-477.

Nestle FO, Di MP, Qin JZ, Nickoloff BJ. Skin immune sentinels in health and disease. Nat Rev Immunol 2009;9:679-691.

Romani N, Flacher V, Tripp CH, Sparber F, Ebner S, Stoitzner P. Targeting skin dendritic cells to improve intradermal vaccination. Curr Top Microbiol Immunol 2012;351:113-138.

Rühl J, Citterio C, Engelmann C, Haigh T, Dzionek A, Dreyer J, et al. Heterologous prime-boost vaccination protects against EBV antigen-expressing lymphomas. J Clin Invest 2019;129:2071-2087.

Schaeffer E, Flacher V, Papageorgiou V, Decossas M, Fauny JD, Kramer M, et al. Dermal CD14(+) Dendritic Cell and Macrophage Infection by Dengue Virus Is Stimulated by Interleukin-4. J Invest Dermatol 2015;135:17431751.

Schlitzer A, McGovern N, Ginhoux F. Dendritic cells and monocyte-derived cells: Two complementary and integrated functional systems. Semin Cell Dev Biol 2015;41:9-22.

Sourisseau M, Schilte C, Casartelli N, Trouillet C, Guivel-Benhassine F, Rudnicka D, et al. Characterization of reemerging chikungunya virus. PLoS Pathog 2007;3:e89.

Stoitzner P, Schaffenrath S, Tripp CH, Reider D, Komenda K, Del FB, et al. Human skin dendritic cells can be targeted in situ by intradermal injection of antibodies against lectin receptors. Exp Dermatol 2014;23:909-915. 


\section{FIGURE LEGENDS}

Figure 1: $C D 14^{+}$dAPCs are infected by Chikungunya virus and selectively capture CHIK-sE2 recombinant envelope protein

(a) HeLa cells, MDdMs, MDDCs and CD14 $4^{+}$dAPCs were exposed to CHIKV at an MOI of 2.5. After $2 \mathrm{~h}$, the cells were washed and cultured for $24 \mathrm{~h}$. The cell supernatant was harvested and viral progeny titrated on AP-61 cells by a plaque assay. (b) Schematic representation of CHIK-SE2 cloned in frame with a SNAP-tag and produced in S2 insect cells. Red residues depict N-glycosylation sites N264, N274 and N346. (c) Covalent coupling to CHIK-sE2 occurs when the SNAP enzymatic moiety reacts with benzylguanine (BG)-coupled AlexaFluor488, generating fluorescent CHIK-sE2-A488. (d) Interaction of CHIK-sE2-A488 with monocytes, MDdMs and MDDCs. The cells were incubated for $30 \mathrm{~min}$ at $37^{\circ} \mathrm{C}$ with $150 \mathrm{nM}$ CHIK-sE2-A488 or left untreated, then analyzed by flow cytometry. Representative histograms are shown. For MDDCs, CHIK-sE2 was preincubated with or without anti-CHIK-sE2 mAb. (e) Relative mean fluorescence intensity (MFI) of CHIK-sE2A488-treated cells vs. untreated cells ( $n=4-6$ donors). (f) Confocal microscopy images of monocytes, MDdMs and MDDCs incubated with CHIK-sE2-A488. Counterstaining of CD14 or HLA-DR allowed visualization of the cell surface. DAPI labels nuclei in blue. Scale bars: $10 \mu \mathrm{m}$. (g) Healthy human skin samples were from donors undergoing plastic surgery. Epidermal cell suspensions were prepared by trypsin digestion. HLA-DR ${ }^{+}$CD1a ${ }^{+}$LCs and HLA-DR- CD1a- keratinocytes were identified by flow cytometry. The dermis was digested by collagenase and $\mathrm{CD} 1 \mathrm{c}^{+} \mathrm{dDC}$ s were isolated by anti-CD1c-coupled magnetic beads. After 2 days of culture, the dermal fibroblasts were identified as HLA-DR- CD14- adherent cells and the non-adherent CD14 ${ }^{+}$dAPCs were isolated by anti-CD14-coupled magnetic beads. $10^{5}$ enriched cells were incubated in serum-free medium for $1 \mathrm{~h}$ at $37^{\circ} \mathrm{C}$ with 150nM CHIK-sE2-A488 or left untreated. Where indicated, CHIK-sE2-A488 was preincubated for 20min with anti-CHIK-sE2 mAb before addition of CD14+ dAPCs. Cells were stained with antibodies against HLA-DR, CD1a, CD14 and CD1c and analyzed by flow cytometry. (h) Relative MFI comparing the fluorescence intensity of CHIK-sE2-A488-treated vs. untreated cells ( $n=3-6$ donors). (i) Confocal microscopy images of CD14 ${ }^{+}$dAPCs exposed to CHIK-sE2-A488 for $30 \mathrm{~min}$ at $37^{\circ} \mathrm{C}$. Cells were labelled for cell-surface HLA-DR, fixed and visualized by fluorescence microscopy. DAPI labels nuclei in blue. Scale bars: $10 \mu \mathrm{m}$. Data were obtained from at least three different donors and are presented as mean \pm SEM. Statistical comparisons: ANOVA followed by posthoc tests (ns: non significant, ${ }^{*} p<0.05,{ }^{* *} p<0.01,{ }^{* * *} p<0.001$ ).

Figure 2: CHIK-sE2 conveys antigenic peptide to $C D 14^{+} d D C s$ for presentation to $C D 4^{+} T$ cells. (a) Whole skin pieces were floated on complete culture medium for 3 days. $10^{5}$ total migratory cells were incubated alone or with $150 \mathrm{nM}$ CHIK-sE2-A488 in serum-free medium for $1 \mathrm{~h}$ at $37^{\circ} \mathrm{C}$. Cells were then stained with antibodies against HLA-DR, Langerin/CD207, DC-SIGN/CD209, and CD14 and analyzed by flow cytometry. (b) Representative histograms of cells treated with CHIK-sE2-A488 or left untreated. (c) Relative MFI comparing 
the fluorescence intensity of CHIK-sE2-A488 vs. untreated cells ( $n=14$ donors). (d) CD14+ dDCs enriched by CD14 MACS were incubated with CHIK-sE2-A488 for 1 h at $37^{\circ} \mathrm{C}$, fixed with $4 \%$ PFA and stained for CD14. Fluorescent CHIK-sE2 in intracellular compartments was visualized by spinning-disk confocal microscopy. Scale bar: $10 \mu \mathrm{m}$. A video rendition is provided as Figure S3. (e) Skin migratory DCs ( $\mathrm{n}=4-10$ donors) were incubated for $30 \mathrm{~min}$ at $37^{\circ} \mathrm{C}$ with $1 \mathrm{mM}$ mannan, $10 \mu \mathrm{g} / \mathrm{mL}$ purified antibodies against DC-SIGN (clones AZN-D1 or $111 \mathrm{H} 2.02$ ), MR (clone 19.2), combinations of anti-MR and anti-DC-SIGN (total concentration $20 \mu \mathrm{g} / \mathrm{mL}$ ) or the corresponding isotype controls. CHIK-sE2-A488 binding was quantified as described above and expressed as $\%$ of MFI obtained for untreated cells. (f) Schematic representation of EBNA1-derived peptide coupling to CHIK-sE2. The selected peptide contains the cognate HLA-DR1-restricted CD4+ $\mathrm{T}$ cell epitope (underlined). (g) EBV-specific T-cell proliferation induced by HLA-DR1-matched skin APCs with and without CHIK-sE2-EBNA1 loading (200nM) was measured by CFSE dilution assays. (h) IFN- $\gamma$ production was assessed by intracellular cytokine staining in proliferating EBNA1-specific $\mathrm{CD} 4^{+} \mathrm{T}$ cells after cognate peptide restimulation of co-cultures with the HLA-DR1-matched skin APCs with and without CHIK-sE2-EBNA1 loading. (i) EBV-specific T-cell proliferation by HLA-DR-mismatched skin APCs was measured as in (g). Representative flow cytometry plots are shown for $(\mathrm{g}, \mathrm{h}, \mathrm{i})$. Data were obtained from at least three different donors and are presented as mean \pm SEM. Statistical comparisons: ANOVA followed by post-hoc tests (nd, not determined, ns: non significant, $\left.{ }^{*} p<0.05, * * p<0.01, * * * p<0.001\right)$. 


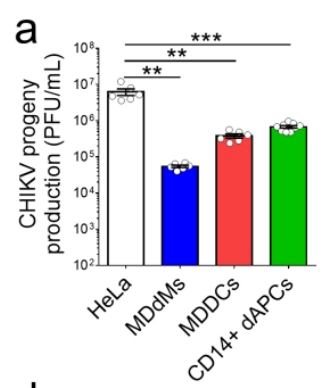

b
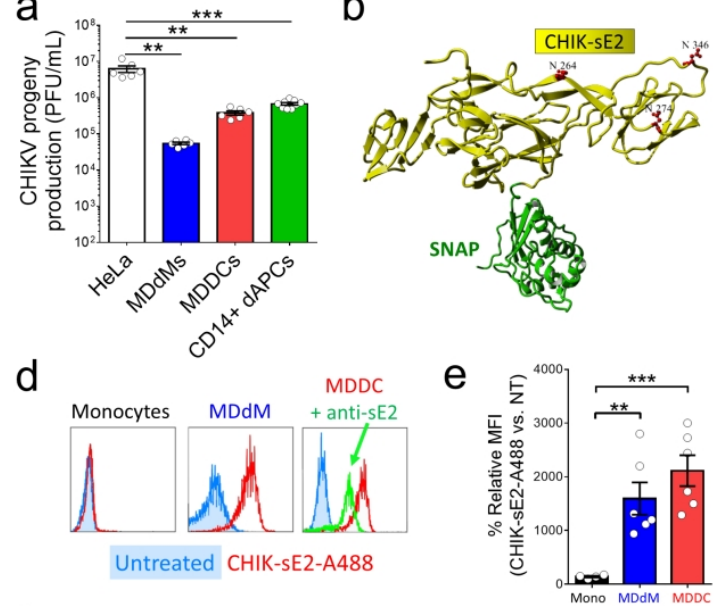

9

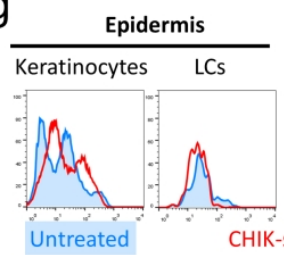

$\mathrm{h}$

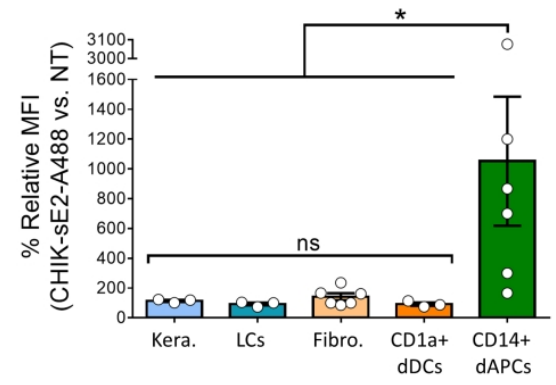

C

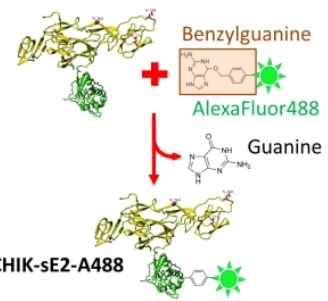

f

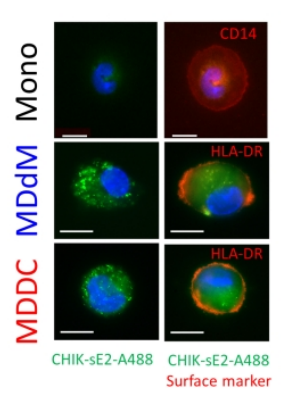

i

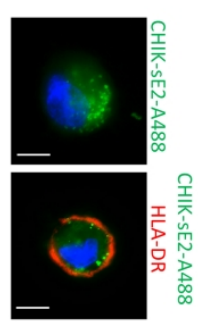

Figure 1

Figure 1: CD14+ dAPCs are infected by Chikungunya virus and selectively capture CHIK-sE2 recombinant envelope protein

(a) HeLa cells, MDdMs, MDDCs and CD14+ dAPCs were exposed to CHIKV at an MOI of 2.5. After $2 \mathrm{~h}$, the cells were washed and cultured for $24 \mathrm{~h}$. The cell supernatant was harvested and viral progeny titrated on AP-61 cells by a plaque assay. (b) Schematic representation of CHIK-sE2 cloned in frame with a SNAP-tag and produced in S2 insect cells. Red residues depict N-glycosylation sites N264, N274 and N346. (c) Covalent coupling to CHIK-sE2 occurs when the SNAP enzymatic moiety reacts with benzylguanine (BG)coupled AlexaFluor488, generating fluorescent CHIK-sE2-A488. (d) Interaction of CHIK-sE2-A488 with

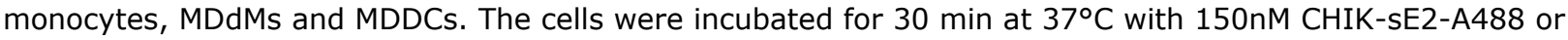
left untreated, then analyzed by flow cytometry. Representative histograms are shown. For MDDCs, CHIKsE2 was preincubated with or without anti-CHIK-sE2 mAb. (e) Relative mean fluorescence intensity (MFI) of CHIK-sE2-A488-treated cells vs. untreated cells ( $n=4-6$ donors). (f) Confocal microscopy images of monocytes, MDdMs and MDDCs incubated with CHIK-sE2-A488. Counterstaining of CD14 or HLA-DR allowed visualization of the cell surface. DAPI labels nuclei in blue. Scale bars: $10 \mu \mathrm{m}$. $(\mathrm{g})$ Healthy human skin 
samples were from donors undergoing plastic surgery. Epidermal cell suspensions were prepared by trypsin digestion. HLA-DR+ CD1a+ LCs and HLA-DR- CD1a- keratinocytes were identified by flow cytometry. The dermis was digested by collagenase and CD1c+ dDCs were isolated by anti-CD1c-coupled magnetic beads. After 2 days of culture, the dermal fibroblasts were identified as HLA-DR- CD14- adherent cells and the nonadherent CD14+ dAPCs were isolated by anti-CD14-coupled magnetic beads. 105 enriched cells were incubated in serum-free medium for $1 \mathrm{~h}$ at $37^{\circ} \mathrm{C}$ with $150 \mathrm{nM} \mathrm{CHIK}-\mathrm{sE} 2-\mathrm{A} 488$ or left untreated. Where indicated, CHIK-sE2-A488 was preincubated for 20min with anti-CHIK-sE2 mAb before addition of CD14+ dAPCs. Cells were stained with antibodies against HLA-DR, CD1a, CD14 and CD1c and analyzed by flow cytometry. (h) Relative MFI comparing the fluorescence intensity of CHIK-sE2-A488-treated vs. untreated cells ( $n=3-6$ donors). (i) Confocal microscopy images of CD14+ dAPCs exposed to CHIK-sE2-A488 for 30 $\min$ at $37^{\circ} \mathrm{C}$. Cells were labelled for cell-surface HLA-DR, fixed and visualized by fluorescence microscopy. DAPI labels nuclei in blue. Scale bars: $10 \mu \mathrm{m}$. Data were obtained from at least three different donors and are presented as mean \pm SEM. Statistical comparisons: ANOVA followed by post-hoc tests (ns: non significant, $* p<0.05, * * p<0.01, * * * p<0.001)$.

$201 \times 288 \mathrm{~mm}(300 \times 300 \mathrm{DPI})$ 

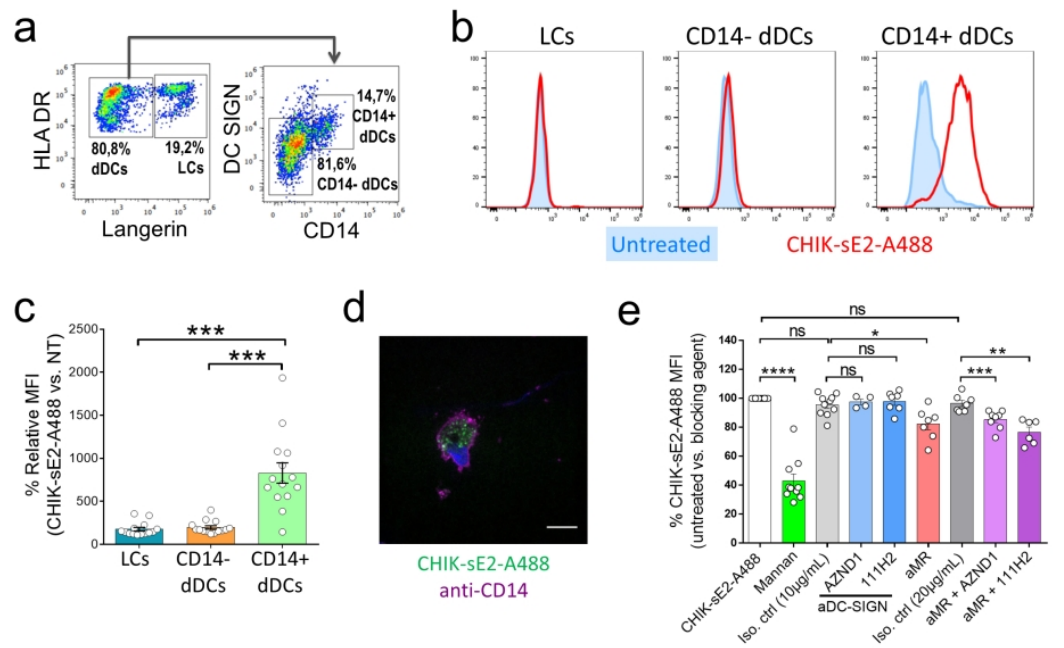

f

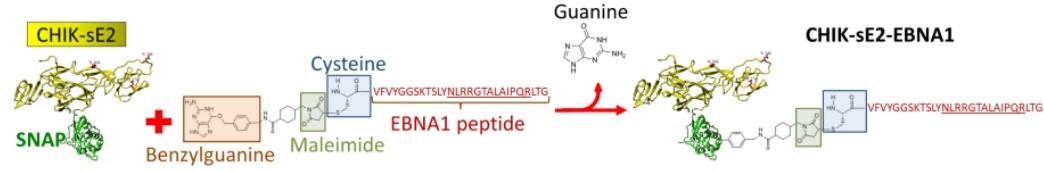

9

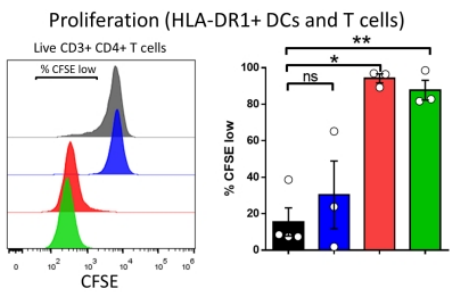

i

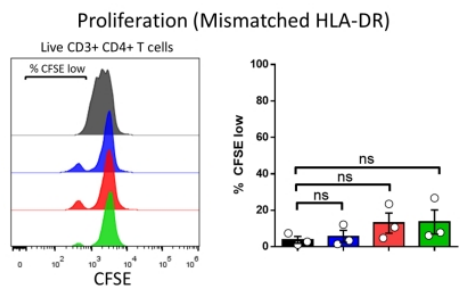

$\mathrm{h}$
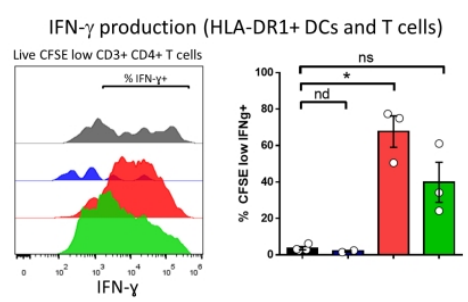

T cells alone

T cells + total skin DCs

T cells + total skin DCs loaded with CHIK-sE2-EBNA1

T cells + CD14+ dDCs loaded with CHIK-sE2-EBNA1

Figure 2

Figure 2: CHIK-sE2 conveys antigenic peptide to CD14+ dDCs for presentation to CD4+ T cells. (a) Whole skin pieces were floated on complete culture medium for 3 days. 105 total migratory cells were incubated alone or with $150 \mathrm{nM}$ CHIK-sE2-A488 in serum-free medium for $1 \mathrm{~h}$ at $37^{\circ} \mathrm{C}$. Cells were then stained with antibodies against HLA-DR, Langerin/CD207, DC-SIGN/CD209, and CD14 and analyzed by flow cytometry.

(b) Representative histograms of cells treated with CHIK-sE2-A488 or left untreated. (c) Relative MFI comparing the fluorescence intensity of CHIK-sE2-A488 vs. untreated cells ( $n=14$ donors). (d) CD14+ dDCs enriched by CD14 MACS were incubated with CHIK-sE2-A488 for $1 \mathrm{~h}$ at $37^{\circ} \mathrm{C}$, fixed with $4 \%$ PFA and stained

for CD14. Fluorescent CHIK-sE2 in intracellular compartments was visualized by spinning-disk confocal microscopy. Scale bar: $10 \mu \mathrm{m}$. A video rendition is provided as Figure S3. (e) Skin migratory DCs $(n=4-10$ donors) were incubated for $30 \mathrm{~min}$ at $37^{\circ} \mathrm{C}$ with $1 \mathrm{mM}$ mannan, $10 \mu \mathrm{g} / \mathrm{mL}$ purified antibodies against DC-SIGN

(clones AZN-D1 or $111 \mathrm{H} 2.02$ ), MR (clone 19.2), combinations of anti-MR and anti-DC-SIGN (total concentration $20 \mu \mathrm{g} / \mathrm{mL}$ ) or the corresponding isotype controls. CHIK-sE2-A488 binding was quantified as described above and expressed as \% of MFI obtained for untreated cells. (f) Schematic representation of EBNA1-derived peptide coupling to CHIK-sE2. The selected peptide contains the cognate HLA-DR1-restricted 
CD4+ T cell epitope (underlined). (g) EBV-specific T-cell proliferation induced by HLA-DR1-matched skin APCs with and without CHIK-sE2-EBNA1 loading (200nM) was measured by CFSE dilution assays. (h) IFN- $\square$ production was assessed by intracellular cytokine staining in proliferating EBNA1-specific CD4+ T cells after cognate peptide restimulation of co-cultures with the HLA-DR1-matched skin APCs with and without CHIKsE2-EBNA1 loading. (i) EBV-specific T-cell proliferation by HLA-DR-mismatched skin APCs was measured as in $(g)$. Representative flow cytometry plots are shown for $(g, h, i)$. Data were obtained from at least three different donors and are presented as mean \pm SEM. Statistical comparisons: ANOVA followed by post-hoc tests (nd, not determined, ns: non significant, $* p<0.05, * * p<0.01, * * * p<0.001$ ).

$201 \times 288 \mathrm{~mm}(300 \times 300$ DPI $)$ 


\section{SUPPLEMENTARY FIGURE LEGENDS}

Figure S1: DC-SIGN and MR are receptors for CHIK-sE2. Affinity of CHIK-SE2 for immobilized MR (a) and DC-SIGN (b) were measured by surface plasmon resonance. Sensorgrams depict association and dissociation to DC-SIGN $(0.1,0.2,0.4,0.8$, and $1.6 \mu \mathrm{M}$ of CHIK-E2) and MR $(3.1,6.3,12.5,25,50$ and 100nM of CHIK-sE2). (c) Constants for the binding assays. The KD for DC-SIGN and MR were 51.1nM and $3.9 \mathrm{nM}$, respectively.

Figure S2: CHIK-sE2 does not promote DC maturation. Immature MDDCs were incubated for $24 \mathrm{~h}$ with $150 \mathrm{nM}$ CHIK-sE2 or $100 \mathrm{ng} / \mathrm{mL}$ LPS. Maturation was quantified by flow cytometry from the MFI of CD86 stainings. Representative histograms are shown. Data were obtained from six different donors and are presented as mean \pm SEM. Statistical comparisons: Wilcoxon tests (ns: non significant, ${ }^{*} p<0.05$ ).

Figure S3: Z-stack video rendition of CD14 ${ }^{+}$dDCs loaded with CHIK-sE2-A488. CD14 ${ }^{+}$dDCs enriched by CD14 MACS were incubated with 150nM CHIK-sE2-A488 in serum-free medium for $1 \mathrm{~h}$ at $37^{\circ} \mathrm{C}$, then fixed with 4\% PFA and counterstained with anti-CD14 to highlight the cell surface (magenta). Fluorescent CHIK-sE2 (green) was visualized in intracellular compartments of targeted cells by spinning-disk confocal microscopy.

\section{SUPPLEMENTARY MATERIAL AND METHODS}

\section{Antibodies and media}

The following antibodies were used for flow cytometry: HLA-DR (clones Tü39 or L243), CD206/MR (19.2), CD209/DC-SIGN (DCN46), CD1a (HI149), CD86 (FUN-1), IFN-ץ (B27), CD3 (UCHT1) from BD, CD14 (MEM-18, Immunotools), CD1C (AD5-8E7) and CD4 (REA623) from Miltenyi Biotec, and CD207/Langerin (DCGM4, Dendritics). Staining buffer was PBS containing $2 \%$ fetal calf serum (FCS; Dutscher) and 2.5mM EDTA (SE buffer). Dead cells were labelled by Fixable Viability Dye-eFluor450 (eBioscience) or Sytox Red dye (Invitrogen). Cells were collected on FACSCalibur (BD) or Gallios (Beckman-Coulter) flow cytometers. Data were analysed with CellQuest (BD), Kaluza (BeckmanCoulter) or FlowJo (Treestar) softwares. Complete medium was RPMI1640 supplemented with $10 \mu \mathrm{g} / \mathrm{mL}$ gentamycin, 100 units $/ \mathrm{mL}$ penicillin, $100 \mu \mathrm{g} / \mathrm{mL}$ streptomycin, $10 \mathrm{mM}$ HEPES (all from Lonza) and $10 \%$ FCS.

\section{Primary cell isolation and culture}

Fresh abdominal skin was obtained from patients undergoing abdominoplasty with written informed consent and institutional review board approval, in agreement with the Helsinki Declaration and French legislation. 
Enzymatic digestion: Subcutaneous fat was removed and the remainder was incubated for $1 \mathrm{~h}$ at $37^{\circ} \mathrm{C}$ in PBS containing 0.5\% trypsin XI (Sigma-Aldrich). The epidermis was then peeled off and agitated in complete medium for $10 \mathrm{~min}$ to liberate LCs and keratinocytes. The dermis was cut into small pieces and digested overnight with $1 \mathrm{mg} / \mathrm{mL}$ collagenase I (Invitrogen) and $50 \mu \mathrm{g} / \mathrm{mL}$ DNAse (Sigma-Aldrich) at $37^{\circ} \mathrm{C}$ and $5 \% \mathrm{CO}_{2}$ in complete medium. The digested dermis was filtered in SE buffer with $50 \mu \mathrm{m}$ cell strainers and dermal CDC2 were isolated by anti-CD1c-coupled magnetic beads (Miltenyi Biotec). Remaining cells were incubated for $48 \mathrm{~h}$ in a $175 \mathrm{~cm}^{2}$ culture flask $\left(50 \times 10^{6}\right.$ cells $/ 50 \mathrm{~mL}$ complete medium). $C D 14^{+}$dAPCs were isolated from the loosely adherent cells using anti-CD14-conjugated magnetic beads (Miltenyi Biotec). After an overnight culture in dermal fibroblast-conditioned culture medium at $10^{6}$ cells $/ \mathrm{mL}, \mathrm{CD} 14^{+} \mathrm{dAPCs}$ were sorted by flow cytometry as CD14+ $\mathrm{HLA}-\mathrm{DR} \mathrm{R}^{\text {low }} \mathrm{CD} 86^{\text {low }}$ cells. Skin explant cultures: $2 \mathrm{~cm}^{2}$ biopsies of $\sim 1 \mathrm{~mm}$ thickness were placed at air-liquid interface onto $40 \mu \mathrm{m}$ cell strainers in $6 \mathrm{~mL}$ complete medium. After 3 days, migratory cells were harvested from the culture supernatant. Resident memory T cells were excluded by selection with anti-CD3 magnetic beads (Miltenyi-Biotec). Where indicated, $\mathrm{CD} 14^{+} \mathrm{dDCs}$ were separated with anti-CD14 magnetic beads (Miltenyi-Biotec).

\section{Monocyte-derived DCs and macrophages}

Monocyte-derived dermal-like macrophages (MDdMs) and MDDCs were generated from blood monocytes as described (Kwan et al., 2008). Briefly, $2 \times 10^{6}$ monocytes, purified from adult blood by counterflow centrifugal elutriation (Etablissement Français du Sang, Strasbourg, France) were cultured in $5 \mathrm{~mL}$ complete medium in the presence of GM-CSF $(50 \mathrm{ng} / \mathrm{mL})$ and IL-4 $(10 \mathrm{ng} / \mathrm{mL})$ for MDDCs, or GMCSF $(25 \mathrm{ng} / \mathrm{mL})$, IL-10 $(25 \mathrm{ng} / \mathrm{mL})$ and M-CSF $(10 \mathrm{ng} / \mathrm{mL})$ for MDdMs. On day 3, the culture was refreshed with $1 \mathrm{~mL}$ of complete medium containing GM-CSF $(50 \mathrm{ng} / \mathrm{mL})$ and IL-4 $(10 \mathrm{ng} / \mathrm{mL})$ for MDDCs, or GMCSF $(12.5 \mathrm{ng} / \mathrm{mL})$ and IL-10 $(12.5 \mathrm{ng} / \mathrm{mL})$ for MDdMs. On day 5, cells were characterized by flow cytometry for expression of CD1a, CD14 and DC-SIGN.

\section{Cell lines}

HeLa and Vero cells were cultured at $37^{\circ} \mathrm{C}$ and $5 \% \mathrm{CO}_{2}$ in DMEM (Invitrogen) supplemented with $10 \%$ FCS and antibiotics. Aedes pseudoscutellaris AP-61 mosquito cells were cultured at $27^{\circ} \mathrm{C}$ in Leibovitz's L-15 medium (Invitrogen) supplemented with 10\% FCS, 1\% tryptose phosphate broth (Eurobio) and antibiotics.

\section{Viral infections}

The CHIKV-06.049 virus strain was isolated from a young adult during the 2006 outbreak of Chikungunya fever in La Réunion island (Schuffenecker et al., 2006). Virus stocks were produced on mosquito AP-61 cells (Brehin et al., 2008) and all infectivity titers were expressed as plaque-forming units on Vero cells. $5 \times 10^{4}$ HeLa cells, CD14 ${ }^{+}$dAPCs, MDdMs and MDDC were exposed to CHIKV at an $\mathrm{MOI}$ of 2.5 , or mock-infected, in RPMI 1640 supplemented with $2 \% \mathrm{FCS}$ for $2 \mathrm{~h}$ at $37^{\circ} \mathrm{C}$. Virus was 
removed by extensive washing and cells were kept for $22 \mathrm{~h}$ in their respective culture media. The supernatants were used for titer analysis by the plaque-forming assay on Vero cells.

\section{Recombinant CHIK-sE2 protein production}

Stably transfected Drosophila melanogaster S2 cell line inducible for the secretion of the ectodomain of CHIKV envelope E2 protein (CHIK-sE2; residues 4-364) were described elsewhere (Aubry et al., 2015). Briefly, a SNAP adaptor sequence (New England Biolabs) was fused in-frame with the C-terminus of recombinant CHIK-sE2, resulting in a chimeric protein that was purified from the supernatants of S2 cells. SNAP is the catalytic site of O6-alkylguanine-DNA-alkyltransférase. 3D rendition of CHIK-sE2SNAP was created with the Yasara software based on the available crystal structures (CHIK-sE2: PDB 3J2W; SNAP-tag: PDB 3KZY).

\section{Surface plasmon resonance}

Surface plasmon resonance experiments were performed using a Biacore 3000. Extracellular domains of DC-SIGN[Lys62-Ala404] and MR[Leu19-Lys1383] (R\&D Systems) were immobilized on a CM5 sensor chip (GE-Healthcare) by injecting $70 \mu \mathrm{L}$ of recombinant proteins $(50 \mu \mathrm{g} / \mathrm{mL}$ in formate buffer, $\mathrm{pH} 4.3$ ) onto the surface activated with $\mathrm{N}$-Ethyl- $\mathrm{N}^{\prime}$-dimethylaminopropyl carbodiimide/N-hydroxysuccinimide. $20 \mu \mathrm{L}$ of ethanolamine hydrochloride, $\mathrm{pH} 8.5$, saturated the free activated sites of the matrix. Biosensor assays were performed with HEPES-buffered-saline (10mM HEPES, 150mM sodium chloride, pH 7.4) containing $0.005 \%$ surfactant $\mathrm{P} 20$ and $5 \mathrm{mM} \mathrm{CaCl} 2$, as running buffer. All binding experiments were carried out at $25^{\circ} \mathrm{C}$ with a constant flow rate of $20 \mu \mathrm{L} / \mathrm{min}$. CHIK-sE2 dissolved in the running buffer was injected for $3 \mathrm{~min}$, followed by a dissociation phase of $3 \mathrm{~min}$. The sensor chip surface was regenerated after each experiment by injecting $10 \mu \mathrm{L}$ EDTA $0.5 \mathrm{M} \mathrm{pH}$ 8.0. The kinetic parameters were calculated using the BIAeval 4.1 software. Analysis was performed using the simple Langmuir binding 1:1 model with separate $\mathrm{ka} / \mathrm{kd}$ (kon/koff). The specific binding profiles were obtained after subtracting the response signal from the control channel and from blank-buffer injection. The fitting to each model was judged by the chi-square value and randomness of residue distribution compared to the theoretical model.

\section{CHIK-sE2 binding studies}

Benzylguanine (BG)-AlexaFluor488 fluorochrome (New England Biolabs) was covalently linked to CHIKSE2 via the SNAP moiety according to the manufacturer's recommendations. $10^{5}$ cells were incubated in $200 \mu \mathrm{L}$ RPMI1640 alone or with $2 \mu \mathrm{g}$ of CHIK-sE2-A488 (final concentration $150 \mathrm{nM}$ ) for $30 \mathrm{~min}$ at $37^{\circ} \mathrm{C}$. The cells were then washed and labelled for cell surface markers in SE buffer and analyzed by flow cytometry. Where indicated, CHIK-sE2 $(1.2 \mu \mathrm{g})$ was pre-incubated for $30 \mathrm{~min}$ at $4^{\circ} \mathrm{C}$ with anti-E2

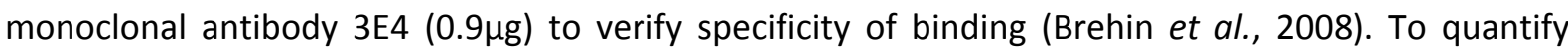
binding efficiency, the mean fluorescent intensity (MFI) of CHIK-sE2-A488-exposed cells was calculated relatively to the MFI of untreated cells, which was set at $100 \%$. For internalization studies by confocal 
microscopy, cells exposed to CHIK-sE2-A488 were washed in SE buffer and counterstained with antibodies against HLA-DR or CD14 for $25 \mathrm{~min}$ on ice. The cells were washed twice, fixed in $4 \%$ formaldehyde for $20 \mathrm{~min}$ at RT, and the secondary goat-anti-mouse AlexaFluor594 antibody (Invitrogen) was added and incubated for $30 \mathrm{~min}$ on ice. After washing, nuclei were labelled with DAPI. Samples were mounted on glass slides in fluorescence mounting medium (Dako). Images taken with Zeiss confocal microscopes LSM510 and Axio Observer Z1 were analyzed with ImageJ. For maturation assays, MDDCs were incubated with 150nM CHIK-sE2 or 100ng/mL ultrapure K12 LPS (Invivogen) for 24h, then CD86 expression was quantified by flow cytometry.

\section{Peptide synthesis}

Peptides were assembled using optimized Fmoc chemistry protocols with a multichannel peptide synthesizer (Neimark and Briand, 1993). Side-chain deprotection and cleavage of peptides from the solid support was performed by treatment with reagent $\mathrm{K}$ ( $82.5 \%$ TFA v/v, $5 \%$ phenol w/v, $5 \%$ water $\mathrm{v} / \mathrm{v}, 5 \%$ thioanisole $\mathrm{v} / \mathrm{v}, 2.5 \%$ 1,2-ethanedithiol $\mathrm{v} / \mathrm{v}$ ) for $150 \mathrm{~min}$ at $20^{\circ} \mathrm{C}$. Peptides were purified by reversed-phase HPLC (RP-HPLC) using a Beckman preparative HPLC system (Gagny, France) on a Nucleosil C18 $(1 \times 30 \mathrm{~cm})$ column, (Macherey Nagel, Hoerdt, France). The elution was achieved with a linear gradient of aqueous $0.1 \%$ TFA and $0.08 \%$ TFA in acetonitrile at a flow rate of $6 \mathrm{~mL} / \mathrm{min}$ with UV detection at $230 \mathrm{~nm}$. The purity of peptides was controlled by analytical RP-HPLC on a Beckman instrument (Gagny, France) with a Nucleosil C18 $5 \mu \mathrm{m}$ column $(150 \times 4.6 \mathrm{~mm})$ using a linear gradient of $0.1 \%$ TFA in water and acetonitrile containing $0.08 \%$ TFA at a flow rate of $1.2 \mathrm{~mL} / \mathrm{min}$. The integrity of each peptide was assessed by LC/MS using a Thermo Finigan LCQ.

The Epstein-Barr virus Nuclear Antigen-1 (EBNA1)-derived peptide (507-535; CVFVYGGSKTSLYNLRRGTALAIPQRLTG) was bound to BG-maleimide (New England Biolabs) by reacting with the thiol group of the N-terminal cysteine residue, before purification by HPLC.

\section{Blocking CHIK binding}

Skin migratory DCs were incubated for $30 \mathrm{~min}$ at $37^{\circ} \mathrm{C}$ with $1 \mathrm{mM}$ mannan (Sigma-Aldrich), $10 \mu \mathrm{g} / \mathrm{mL}$ purified antibodies against DC-SIGN (clone AZN-D1, Dr. Teunis Geijtenbeek, Amsterdam, NL (Geijtenbeek et al., 2000); clone 111H2.02, Dendritics, France (Canard et al., 2011)), MR (clone 19.2, $\mathrm{BD}$ ), combinations of anti-MR and anti-DC-SIGN (total concentration $20 \mu \mathrm{g} / \mathrm{mL}$ ) or the corresponding isotype controls. Then, $150 \mathrm{nM}$ CHIK-sE2-A488 was added for $1 \mathrm{~h}$ and its binding was quantified by flow cytometry.

\section{Expansion of EBNA1-specific CD4+ T memory cells}

Allogeneic PBMCs were purified from buffy coats of healthy blood donors (EFS) by Ficoll gradient (Sigma-Aldrich). $5 \times 10^{3}$ EBNA1-specific CD4 ${ }^{+}$T cell (CK-NLR clone, HLA-DR1-restricted) cells/well were cocultured with $10^{5}$ irradiated PBMCs (3000rad) and $2 \times 10^{3}$ irradiated, EBV-infected autologous B-LCL (20000rad) in RPMI1640 supplemented with 8\% PHS (pooled human sera, Corning), 150U/mL IL-2 
(Novartis), $1 \mu \mathrm{g} / \mathrm{mL}$ Phytohemagglutinin-L (Sigma-Aldrich), and gentamicin. After 5 days, half of the medium was refreshed with RPMI $1640+8 \%$ PHS + gentamycin $+450 \mathrm{U} / \mathrm{mL}$ IL-2. Cells were monitored daily and split into the same medium and new 96 well-plates if necessary, until freezing at day 14 (Rühl et al., 2019).

\section{Antigen presentation assays}

Genomic DNA was extracted from 40-80mg of skin using Nucleospin columns (Macherey-Nagel) and HLA haplotype of donors was determined with SSP HLA DQDR Combitray kit (Olerup). BG-EBNA1 peptide (250pmol) was coupled to CHIK-sE2 (200pmol) via the SNAP moiety in PBS. CHIK-sE2-EBNA1 (200nM) was incubated with $5 \times 10^{5}$ migratory skin DCs or CD14 ${ }^{+}$dDCs for $1 \mathrm{~h}$ in RPMI1640 medium. $10^{4}$ T-cell depleted, CHIK-EBNA1-loaded skin DCs or CD14+ dDCs from HLA-DR1 donors were cocultured in $200 \mu \mathrm{L}$ RPMI1640 + 8\% PHS in 96-well U-bottom plates with $5 \times 10^{4}$ EBNA1-specific $\mathrm{CD}^{+}{ }^{+} \mathrm{T}$ cells previously labelled with $0.5 \mu \mathrm{M}$ carboxyfluorescein succinimidyl ester (CFSE). After 5 days, T cells were restimulated $6 \mathrm{~h}$ with EBNA1 peptide $(1 \mu \mathrm{g} / \mathrm{mL})$, including $4 \mathrm{~h}$ with $10 \mu \mathrm{g} / \mathrm{mL}$ brefeldin $\mathrm{A}$ and $1 \mu \mathrm{M}$ monensin (BD). $T$ cells were stained for $C D 3$ and $C D 4$, then fixed and permeabilized with $B D$ Cytofix/Cytoperm Kit, stained with anti-IFN- $\nu$ antibody and analyzed by flow cytometry. T-cell proliferation was determined by CFSE dilution.

\section{Statistical analyses}

Data from different experimental conditions were tested by ANOVA, followed by relevant post-hoc multiple comparisons or by Wilcoxon tests, performed with Prism 6.0 software (GraphPad). Values of p below 0.05 were considered significant. 


\section{REFERENCES}

Aubry M, Finke J, Teissier A, Roche C, Broult J, Paulous S, et al. Silent Circulation of Ross River Virus in French Polynesia. Int J Infect Dis 2015;37:19-24.

Brehin AC, Rubrecht L, Navarro-Sanchez ME, Marechal V, Frenkiel MP, Lapalud P, et al. Production and characterization of mouse monoclonal antibodies reactive to Chikungunya envelope E2 glycoprotein. Virology 2008;371:185-195.

Canard B, Vachon H, Fontaine T, Pin JJ, Paul S, Genin C, et al. Generation of anti-DC-SIGN monoclonal antibodies capable of blocking HIV-1 gp120 binding and reactive on formalin-fixed tissue. Immunol Lett 2011;135:165-172.

Geijtenbeek TBH, Torensma R, Van Vliet SJ, Van Duijnhoven GCF, Adema GJ, Van Kooyk Y, et al. Identification of DC-SIGN, a novel dendritic cell-specific ICAM-3 receptor that supports primary immune responses. Cell 2000;100:575-585.

Kwan WH, Navarro-Sanchez E, Dumortier H, Decossas M, Vachon H, dos Santos FB, et al. Dermal-type macrophages expressing CD209/DC-SIGN show inherent resistance to dengue virus growth. PLoS NegI Trop Dis 2008;2:e311.

Neimark J, Briand JP. Development of a fully automated multichannel peptide synthesizer with integrated TFA cleavage capability. Pept Res 1993;6:219-228.

Rühl J, Citterio C, Engelmann C, Haigh T, Dzionek A, Dreyer J, et al. Heterologous prime-boost vaccination protects against EBV antigen-expressing lymphomas. J Clin Invest 2019;129:2071-2087.

Schuffenecker I, Iteman I, Michault A, Murri S, Frangeul L, Vaney MC, et al. Genome microevolution of chikungunya viruses causing the Indian Ocean outbreak. PLoS Med 2006;3:e263. 


\section{a}

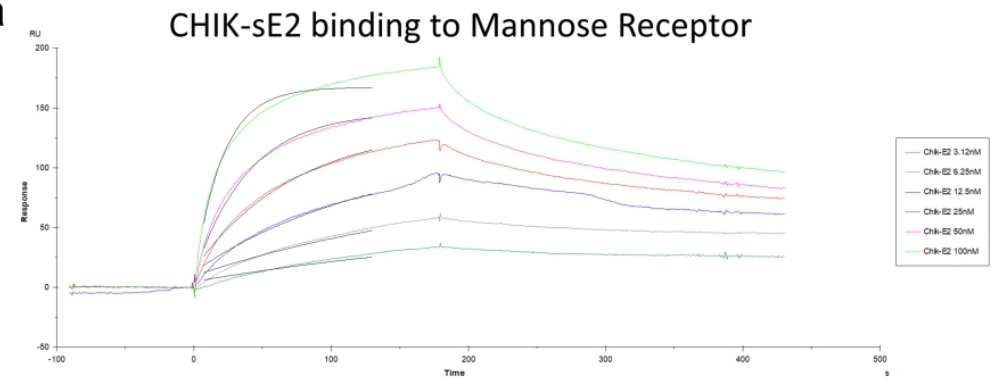

b

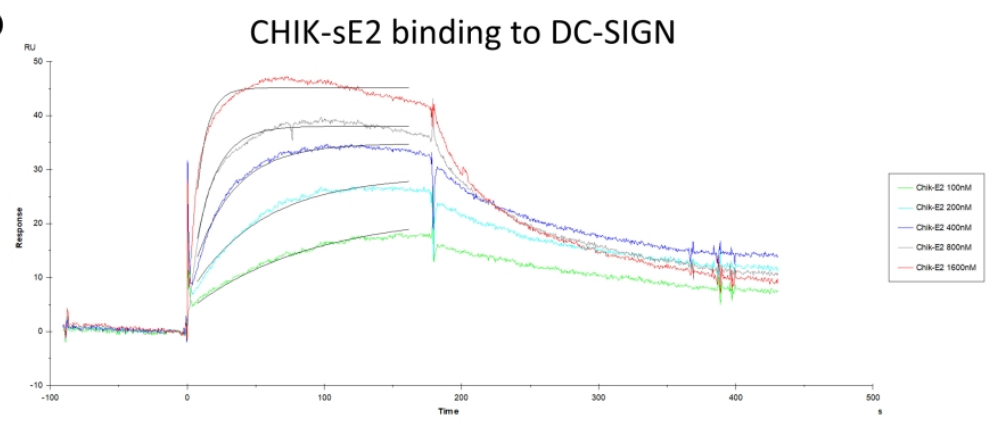

C

\begin{tabular}{|c|c|c|c|c|c|c|}
\hline & $\mathbf{k a}(\mathbf{1} / \mathbf{M s})$ & $\mathbf{k d}(\mathbf{1} / \mathbf{s})$ & $\mathbf{R m a x}(\mathbf{R U})$ & $\mathrm{KA}(\mathbf{1} / \mathrm{M})$ & $\mathrm{KD}(\mathbf{n M})$ & Chi $^{\mathbf{2}}$ \\
\hline MR & $4.72 \times 10^{5}$ & $1.83 \times 10^{-3}$ & 130.0 & $2.58 \times 10^{8}$ & 3.88 & 10.20 \\
\hline DC-SIGN & $7.91 \times 10^{4}$ & $4.04 \times 10^{-3}$ & 29.7 & $1.96 \times 10^{7}$ & 51.10 & 2.44 \\
\hline
\end{tabular}

\section{Supplementary Figure S1}

Figure S1: DC-SIGN and MR are receptors for CHIK-sE2. Affinity of CHIK-sE2 for immobilized MR (a) and DC-SIGN (b) were measured by surface plasmon resonance. Sensorgrams depict association and dissociation to DC-SIGN $(0.1,0.2,0.4,0.8$, and $1.6 \mu \mathrm{M}$ of CHIK-E2) and MR $(3.1,6.3,12.5,25,50$ and $100 \mathrm{nM}$ of CHIK-sE2). (c) Constants for the binding assays. The KD for DC-SIGN and MR were $51.1 \mathrm{nM}$ and 3.9nM, respectively.

$201 \times 288 \mathrm{~mm}(300 \times 300 \mathrm{DPI})$ 

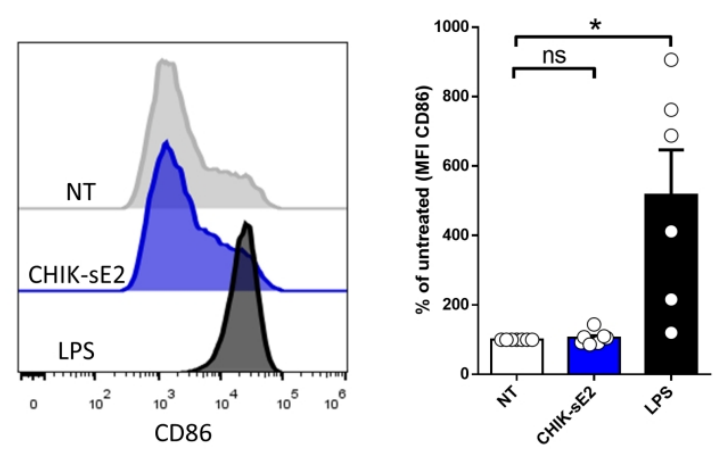

Supplementary Figure S2

Figure S2: CHIK-sE2 does not promote DC maturation. Immature MDDCs were incubated for $24 \mathrm{~h}$ with $150 \mathrm{nM}$ CHIK-sE2 or $100 \mathrm{ng} / \mathrm{mL}$ LPS. Maturation was quantified by flow cytometry from the MFI of CD86 stainings. Representative histograms are shown. Data were obtained from six different donors and are presented as mean \pm SEM. Statistical comparisons: Wilcoxon tests ( $n s$ : non significant, ${ }^{*} p<0.05$ ).

$$
201 \times 288 \mathrm{~mm}(300 \times 300 \text { DPI })
$$


NOTICE TO EDITORS AND REVIEWERS:

The authors have provided 1 supplementary video file. Please request this video file from the editorial office by emailing jidoffice2@sidnet.org. 
Chikungunya virus envelope protein E2 provides a vector for targeted antigen delivery to human dermal CD14+ dendritic cells.

Adrien Brulefert ${ }^{1}$, Melanie Kraemer ${ }^{1}$, Marie Cumin ${ }^{1}$, Amandine Selle ${ }^{1}$, Astrid Hoste ${ }^{1}$, Hans-Henrik Gad², Julia Rühl$^{3}$, Jean-Baptiste Madinier ${ }^{1}$, Olivier Chaloin ${ }^{1}$, Christian Münz ${ }^{3}$, Philippe Desprès ${ }^{2,4}$, Christopher George Mueller $^{1}$, Vincent Flacher ${ }^{1 *}$

1. Laboratory CNRS I²T/UPR3572 Immunology, Immunopathology and Therapeutic Chemistry, Strasbourg Drug Discovery and Development Institute (IMS), Institut de Biologie Moléculaire et Cellulaire, Strasbourg, France

2. Unité Interactions Moléculaires Flavivirus-Hôtes, Institut Pasteur, Paris, France.

3. Viral Immunobiology, Institute of Experimental Immunology, University of Zürich, Zürich, Switzerland.

4. Present address: Université de La Réunion, INSERM U1187, CNRS UMR 9192, IRD UMR 249, Unité Mixte Processus Infectieux en Milieu Insulaire Tropical, Plateforme Technologique CYROI, 97491 Sainte-Clotilde, La Réunion, France.

*Correspondence: v.flacher@ibmc-cnrs.unistra.fr

1005 words 


\section{TO THE EDITOR}

Therapeutic modulation of human cutaneous antigen-presenting cells (APCs) that control the fate of T cells represents an interesting option for immunotherapy. As they capture glycosylated proteins via C-type lectins, lectin-targeting antibodies have been used to specifically convey antigens to APCs (Romani et al., 2012;Stoitzner et al., 2014;Kastenmuller et al., 2014). Beyond potential therapeutic applications, APCtargeting strategies can be harnessed to acquire fundamental knowledge about their function (Flacher et al., 2014). In human skin, epidermal Langerhans cells (LCs), CD1c dermal type-2 conventional dendritic cells (cDC2) and CD141 ${ }^{+}$dermal CDC1 (Nestle et al., 2009;Schlitzer et al., 2015) can migrate to lymph nodes and stimulate T cells. Dermal APCs (dAPCs) include two CD14+ subsets that express the lectins Mannose Receptor (MR)/CD206 and DC-specific intracellular adhesion molecules-3 grabbing non-integrin (DC-SIGN)/CD209 (Klechevsky et al., 2008;McGovern et al., 2014). During skin explant culture, CD14+ dermal DCs (dDCs) migrate into the supernatant, whereas sessile dermal macrophages require enzymatic digestion to be isolated. Still, CD14+ dDCs and dermal macrophages display strong similarities (McGovern et al., 2014). They are both considered permanent dermal residents that exert local functions in pathogen capture (e.g. arthropod-borne viruses) (Kwan et al., 2008;Schaeffer et al., 2015) and may interact with skin-resident memory T cells (TRM) (Clark, 2015). Subset differences might reflect their origin, i.e. monocyte-derived dDCs vs. embryonically seeded resident macrophages (Ginhoux and Guilliams, 2016;McGovern et al., 2014). Because tools to specifically target $\mathrm{CD} 14^{+} \mathrm{dAPC}$ remain scarce (Duinkerken et al., 2019), we propose here a novel approach based on recombinant arboviral envelope proteins.

Chikungunya virus (CHIKV) of the flavivirus genus of Togaviridae family is introduced into the dermis during blood meals of mosquitoes. CHIKV infection triggers high fever, skin rash and persistent arthralgia. Our finding that CD14+ dAPCs are preferentially infected by Dengue virus (Kwan et al., 2008;Schaeffer et al., 2015) prompted the idea that they may represent an immediate CHIKV target. CD14+ dAPCs isolated from human dermis, monocyte-derived DCs (MDDCs) and dermal-like macrophages (MDdMs) were exposed to CHIKV-06.049 strain (MOI=2.5) and compared with HeLa cells, in which vigorous viral replication is expected (Sourisseau et al., 2007). CD14 ${ }^{+}$dAPCs and their monocyte-derived equivalent MDdMs were permissive CHIKV target cells, as well as MDDCs, which resemble the inflammatory DCs recruited to the site of infection

\section{(Figure 1A).}

We hypothesized that APCs express endocytic receptors of viral envelope proteins. CHIK-sE2, a soluble form of CHIKV envelope glycoprotein E2, was fused to the SNAP proprietary enzyme and produced in insect cells to retain specific $\mathrm{N}$-glycosylations that may be recognized by lectins (Figure 1B). In surface plasmon resonance assays, CHIK-sE2 displayed high affinity for MR and DC-SIGN (Figure S1A/B), with dissociation constants of $3.9 \mathrm{nM}$ and $51.1 \mathrm{nM}$, respectively (Figure S1C). CHIK-sE2 was then covalently conjugated via its enzymatic SNAP moiety to AlexaFluor488 fluorochrome (Figure 1C). Since MDdMs and MDDCs, but not 
monocytes, express high levels of lectins (Kwan et al., 2008), we assessed their binding of the recombinant viral glycoprotein. CHIK-sE2-A488 only labelled MDdMs and MDDCs, and an anti-CHIK-sE2 antibody diminished binding to MDDCs (Figure 1D/E). Moreover, fluorescence microscopy revealed intracellular vesicles containing CHIK-sE2-A488 within MDdMs and MDDCs, but not in monocytes (Figure 1F). Incubation with CHIK-sE2 had no influence on MDDC maturation, estimated by CD86 expression (Figure S2).

To evaluate whether CHIK-sE2 can target CD14 ${ }^{+}$dAPCs within the skin, we compared its capture by different primary cutaneous APCs and non-hematopoietic cells. Epidermal cell suspensions, fibroblasts or purified $\mathrm{CD}_{14}{ }^{+} \mathrm{dAPCs}$ and $\mathrm{CD} 1 \mathrm{c}^{+} \mathrm{dDCs}$ were incubated with CHIK-sE2-A488 and binding efficacy was measured by flow cytometry. CD14+ $\mathrm{dAPCS}$ displayed clear binding of CHIK-sE2-A488, as opposed to keratinocytes, LCs, fibroblasts and CD1c dDCs (Figure 1G). Anti-CHIK-sE2 mAb inhibited the interaction with CD14 ${ }^{+}$dAPCs. Quantitative analyses on skin samples from different individuals confirmed that only CD14 dAPCs could efficiently capture CHIK-sE2-A488 (Figure 1 H). CHIK-sE2-A488 was detected as condensed spots in the cytoplasm of $\mathrm{CD} 14^{+} \mathrm{dAPC}$, reflecting endocytic uptake (Figure 11).

We next tested whether CHIK-sE2 could be used to convey antigens to CD14+ $d D C s$, which stimulate T cells more potently than macrophages (McGovern et al., 2014). To assess binding to $C D 14^{+} \mathrm{dDCs}$ in comparison with the other skin DC subsets, we cultured skin explants for 3 days and collected LCs, CD14 $d D C s$ and $C D 14^{+} d D C s$ from the supernatant (Figure 2A). When the cell suspension was exposed to CHIK-sE2A488, only $C D 14^{+} d D C s$ significantly captured (Figure 2B/C) and internalized (Figure 2D, Video S3) the fluorescent protein. Mannan potently decreased CHIK-sE2-A488 binding, and inhibition assays using antilectin mAb suggested that MR is a more efficient CHIK-sE2 receptor than DC-SIGN (Figure 2E).

To address antigen presentation, we used a $\mathrm{CD}^{+}{ }^{+}$T-cell clone with defined specificity for HLA-DR1restricted antigenic peptide of the Epstein-Barr virus Nuclear Antigen-1 (EBNA1) (Rühl et al., 2019). Total migratory APCs or CD14 $4^{+}$dDCs from HLA-DR1 ${ }^{+}$skin explants (5 donors out of 34 ) were loaded with CHIK-sE2 conjugated to EBNA1 peptide (Figure 2F). APCs were then cocultured with CFSE-labelled, EBNA1-specific $\mathrm{CD}^{+} \mathrm{T}$ cells. After 5 days, proliferation and intracellular IFN- $\gamma$ production were quantified by flow cytometry. CHIK-sE2-EBNA1-exposed APCs from HLA-DR1 donors strongly enhanced proliferation (Figure 2G) and triggered IFN- $\gamma$ production (Figure $\mathbf{2 H}$ ) of EBNA1-specific $\mathrm{T}$ cells. However, $\mathrm{T}$ cells did not proliferate when cultured with mismatched, non-HLA-DR1 APCs (Figure 2I). Targeting purified CD14+ dDCs with CHIK-sE2EBNA1 was sufficient to promote EBNA1-specific T-cell proliferation. Yet, IFN- $\gamma$ production was not significantly enhanced, possibly because CD14 ${ }^{+} \mathrm{dDC}$ s produce anti-inflammatory IL-10 and display low CD86 expression (Klechevsky et al., 2008).

Cutaneous TRM are derived from effector T cells that colonize the skin to mediate adaptive immune responses (Clark, 2015). CD14+ dAPCs might be critically involved during secondary exposure to cognate antigen because they also permanently reside in the skin and can reactivate memory $T$ cells (McGovern et 
al., 2014). CD14 ${ }^{+}$dAPCs targeting by CHIK-sE2 could be particularly useful to investigate and influence these interactions for therapeutic purposes. Moreover, our work suggests that arboviruses may represent a natural reservoir of molecules targeting different skin APCs. They may be derived into a specific delivery system for antigens but also adjuvants, silencing RNA or toxins, thereby harnessing the properties of skin APCs for immunotherapy or vaccination.

\section{ORCID iD}

Adrien Brulefert: https://orcid.org/0000-0002-3307-4298

Melanie Kraemer : https://orcid.org/0000-0002-0208-7029

Marie Cumin : https://orcid.org/0000-0002-4776-8231

Amandine Selle: https://orcid.org/0000-0002-9057-8580

Astrid Hoste: https://orcid.org/0000-0002-3531-1285

Hans-Henrik Gad: https://orcid.org/0000-0001-8449-1115

Julia Rühl: https://orcid.org/0000-0001-8385-4367

Jean-Baptiste Madinier: https://orcid.org/0000-0001-9503-2619

Olivier Chaloin: https://orcid.org/0000-0003-3045-4452

Christian Münz: https://orcid.org/0000-0001-6419-1940

Philippe Desprès: https://orcid.org/0000-0001-8926-4050

Christopher George Mueller: https://orcid.org/0000-0002-4119-2729

Vincent Flacher: https://orcid.org/0000-0002-8648-2615

\section{Data availability}

No datasets were generated or analyzed during the current study.

\section{Conflict of Interest Statement}

The authors declare no conflict of interest.

\section{Acknowledgements}

PD, CGM and VF designed the research. JR and CM provided EBNA-1-specific T cell lines and methods for antigen-specific assays. JBM and OC produced antigenic peptides. OC performed surface plasmon resonance assays. $A B, M K, M C, A S, A H, H H G, C G M$ and VF performed experiments. $A B, M K, C G M$ and $V F$ analyzed the data. $A B, P D, C G M$ and VF wrote the manuscript. The authors wish to thank Dr. Teunis Geijtenbeek (University of Amsterdam, NL) for anti-DC-SIGN mAb clone AZN-D1, as well as Drs. Sylvie Tourne and Henri De La Salle (Etablissement Français du Sang, Strasbourg, France) for their advice on HLA typing assays. This 
work was supported by the European Union Marie-Slodowska-Curie Career Integration Grant of Dr. V. Flacher (PCIG12-GA-2012-334011 - DERMACRO) and by the Agence Nationale pour la Recherche (Program “Investissements d'Avenir," ANR-10-LABX-0034 MEDALIS and ANR-11-EQPX-022).

\section{CRediT statement}

Conceptualization: PD, CGM, VF; Data Curation: AB, CGM, VF; Formal Analysis: AB, CGM, VF; Funding Acquisition: CGM, VF; Investigation: AB, MK, MC, AS, AH, HHG, CGM, VF; Methodology: OC, CM, PD, CGM, VF; Project administration: CGM, VF; Resources: JBM, OC, PD, JR, CM, PD; Supervision: PD, CGM, VF; Validation: $A B, M K, M C, A S, A H, H H G$, JR, JBM, OC, CM, PD, CGM, VF; Visualization: AB, OC, CGM, VF; Writing - Original Draft Preparation: AB, PD, CGM, VF; Writing - Review and Editing: AB, PD, CGM, VF. 


\section{REFERENCES}

Clark RA. Resident memory T cells in human health and disease. Sci Transl Med 2015;7:269rv1.

Duinkerken S, Horrevorts SK, Kalay H, Ambrosini M, Rutte L, De Gruijl TD, et al. Glyco-Dendrimers as Intradermal Anti-Tumor Vaccine Targeting Multiple Skin DC Subsets. Theranostics 2019;9:5797-5809.

Flacher V, Tripp CH, Mairhofer DG, Steinman RM, Stoitzner P, Idoyaga J, et al. Murine Langerin+ dermal dendritic cells prime CD8+ $T$ cells while Langerhans cells induce cross-tolerance. EMBO Mol Med 2014;6:1191-1204.

Ginhoux F, Guilliams M. Tissue-Resident Macrophage Ontogeny and Homeostasis. Immunity 2016;44:439449.

Kastenmuller W, Kastenmuller K, Kurts C, Seder RA. Dendritic cell-targeted vaccines--hope or hype? Nat Rev Immunol 2014;14:705-711.

Klechevsky E, Morita R, Liu M, Cao Y, Coquery S, Thompson-Snipes L, et al. Functional specializations of human epidermal Langerhans cells and CD14+ dermal dendritic cells. Immunity 2008;29:497-510.

Kwan WH, Navarro-Sanchez E, Dumortier H, Decossas M, Vachon H, dos Santos FB, et al. Dermal-type macrophages expressing CD209/DC-SIGN show inherent resistance to dengue virus growth. PLoS NegI Trop Dis 2008;2:e311.

McGovern N, Schlitzer A, Gunawan M, Jardine L, Shin A, Poyner E, et al. Human dermal CD14(+) cells are a transient population of monocyte-derived macrophages. Immunity 2014;41:465-477.

Nestle FO, Di MP, Qin JZ, Nickoloff BJ. Skin immune sentinels in health and disease. Nat Rev Immunol 2009;9:679-691.

Romani N, Flacher V, Tripp CH, Sparber F, Ebner S, Stoitzner P. Targeting skin dendritic cells to improve intradermal vaccination. Curr Top Microbiol Immunol 2012;351:113-138.

Rühl J, Citterio C, Engelmann C, Haigh T, Dzionek A, Dreyer J, et al. Heterologous prime-boost vaccination protects against EBV antigen-expressing lymphomas. J Clin Invest 2019;129:2071-2087.

Schaeffer E, Flacher V, Papageorgiou V, Decossas M, Fauny JD, Kramer M, et al. Dermal CD14(+) Dendritic Cell and Macrophage Infection by Dengue Virus Is Stimulated by Interleukin-4. J Invest Dermatol 2015;135:1743-1751.

Schlitzer A, McGovern N, Ginhoux F. Dendritic cells and monocyte-derived cells: Two complementary and integrated functional systems. Semin Cell Dev Biol 2015;41:9-22.

Sourisseau M, Schilte C, Casartelli N, Trouillet C, Guivel-Benhassine F, Rudnicka D, et al. Characterization of reemerging chikungunya virus. PLoS Pathog 2007;3:e89.

Stoitzner P, Schaffenrath S, Tripp CH, Reider D, Komenda K, Del FB, et al. Human skin dendritic cells can be targeted in situ by intradermal injection of antibodies against lectin receptors. Exp Dermatol 2014;23:909915. 


\section{FIGURE LEGENDS}

Figure 1: $\mathrm{CD} 14^{+}$dAPCs are infected by Chikungunya virus and selectively capture CHIK-sE2 recombinant envelope protein

(A) HeLa cells, MDdMs, MDDCs and CD14 ${ }^{+}$dAPCs were exposed to CHIKV at an MOI of 2.5. After 2h, the cells were washed and cultured for $24 \mathrm{~h}$. The cell supernatant was harvested and viral progeny titrated on AP-61 cells by a plaque assay. (B) Schematic representation of CHIK-sE2 cloned in frame with a SNAP-tag and produced in S2 insect cells. Red residues depict N-glycosylation sites N264, N274 and N346. (C) Covalent coupling to CHIK-sE2 occurs when the SNAP enzymatic moiety reacts with benzylguanine (BG)-coupled AlexaFluor488, generating fluorescent CHIK-sE2-A488. (D) Interaction of CHIK-sE2-A488 with monocytes,

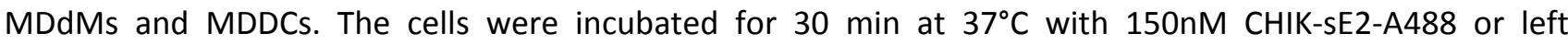
untreated, then analyzed by flow cytometry. Representative histograms are shown. For MDDCs, CHIK-sE2 was preincubated with or without anti-CHIK-sE2 mAb. (E) Relative mean fluorescence intensity (MFI) of CHIK-sE2-A488-treated cells vs. untreated cells ( $n=4-6$ donors). (F) Confocal microscopy images of monocytes, MDdMs and MDDCs incubated with CHIK-sE2-A488. Counterstaining of CD14 or HLA-DR allowed visualization of the cell surface. DAPI labels nuclei in blue. Scale bars: 10 10 m. (G) Healthy human skin samples were from donors undergoing plastic surgery. Epidermal cell suspensions were prepared by trypsin digestion. HLA-DR ${ }^{+}$CD1a+ ${ }^{+}$CS and HLA-DR- CD1a- keratinocytes were identified by flow cytometry. The dermis was digested by collagenase and $C D 1 c^{+} d D C s$ were isolated by anti-CD1c-coupled magnetic beads. After 2 days of culture, the dermal fibroblasts were identified as HLA-DR- CD14- adherent cells and the nonadherent $\mathrm{CD}_{1} 4^{+} \mathrm{dAPCs}$ were isolated by anti-CD14-coupled magnetic beads. $10^{5}$ enriched cells were incubated in serum-free medium for $1 \mathrm{~h}$ at $37^{\circ} \mathrm{C}$ with $150 \mathrm{nM}$ CHIK-sE2-A488 or left untreated. Where indicated, CHIK-sE2-A488 was preincubated for $20 \mathrm{~min}$ with anti-CHIK-sE2 $\mathrm{mAb}$ before addition of CD14 ${ }^{+}$ dAPCs. Cells were stained with antibodies against HLA-DR, CD1a, CD14 and CD1c and analyzed by flow cytometry. (H) Relative MFI comparing the fluorescence intensity of CHIK-sE2-A488-treated vs. untreated cells ( $n=3-6$ donors). (I) Confocal microscopy images of CD14+ dAPCs exposed to CHIK-sE2-A488 for 30 min at $37^{\circ} \mathrm{C}$. Cells were labelled for cell-surface HLA-DR, fixed and visualized by fluorescence microscopy. DAPI labels nuclei in blue. Scale bars: $10 \mu \mathrm{m}$. Data were obtained from at least three different donors and are presented as mean \pm SEM. Statistical comparisons: ANOVA followed by post-hoc tests (ns: non significant, $\left.{ }^{*} \mathrm{p}<0.05,{ }^{* *} \mathrm{p}<0.01, * * * \mathrm{p}<0.001\right)$.

Figure 2: CHIK-sE2 conveys antigenic peptide to $C D 14^{+} d D C$ s for presentation to $C D 4^{+} T$ cells. (A) Whole skin pieces were floated on complete culture medium for 3 days. $10^{5}$ total migratory cells were incubated alone or with $150 \mathrm{nM}$ CHIK-sE2-A488 in serum-free medium for $1 \mathrm{~h}$ at $37^{\circ} \mathrm{C}$. Cells were then stained with antibodies against HLA-DR, Langerin/CD207, DC-SIGN/CD209, and CD14 and analyzed by flow cytometry. (B) 
Representative histograms of cells treated with CHIK-sE2-A488 or left untreated. (C) Relative MFI comparing the fluorescence intensity of CHIK-sE2-A488 vs. untreated cells ( $n=14$ donors). (D) CD14 ${ }^{+}$dDCs enriched by CD14 MACS were incubated with CHIK-sE2-A488 for 1 h at $37^{\circ} \mathrm{C}$, fixed with $4 \%$ PFA and stained for CD14. Fluorescent CHIK-sE2 in intracellular compartments was visualized by spinning-disk confocal microscopy. A video rendition is provided as Figure S3. (E) Skin migratory DCs ( $n=4-10$ donors) were incubated for 30min at $37^{\circ} \mathrm{C}$ with $1 \mathrm{mM}$ mannan, $10 \mu \mathrm{g} / \mathrm{mL}$ purified antibodies against DC-SIGN (clones AZN-D1 or $111 \mathrm{H} 2.02$ ), MR (clone 19.2), combinations of anti-MR and anti-DC-SIGN (total concentration $20 \mu \mathrm{g} / \mathrm{mL}$ ) or the corresponding isotype controls. CHIK-sE2-A488 binding was quantified as described above and expressed as \% of MFI obtained for untreated cells. (F) Schematic representation of EBNA1-derived peptide coupling to CHIK-sE2. The selected peptide contains the cognate HLA-DR1-restricted CD4+ ${ }^{+}$cell epitope (underlined). (G) EBVspecific T-cell proliferation induced by HLA-DR1-matched skin APCs with and without CHIK-sE2-EBNA1 loading (200nM) was measured by CFSE dilution assays. (H) IFN- $\gamma$ production was assessed by intracellular cytokine staining in proliferating EBNA1-specific $C D 4^{+} \mathrm{T}$ cells after cognate peptide restimulation of cocultures with the HLA-DR1-matched skin APCs with and without CHIK-sE2-EBNA1 loading. (I) EBV-specific Tcell proliferation by HLA-DR-mismatched skin APCs was measured as in (G). Representative flow cytometry plots are shown for $\mathrm{G}, \mathrm{H}, \mathrm{I}$. Data were obtained from at least three different donors and are presented as mean \pm SEM. Statistical comparisons: ANOVA followed by post-hoc tests (nd, not determined, ns: non significant, $\left.{ }^{*} p<0.05,{ }^{* *} p<0.01, * * * p<0.001\right)$. 

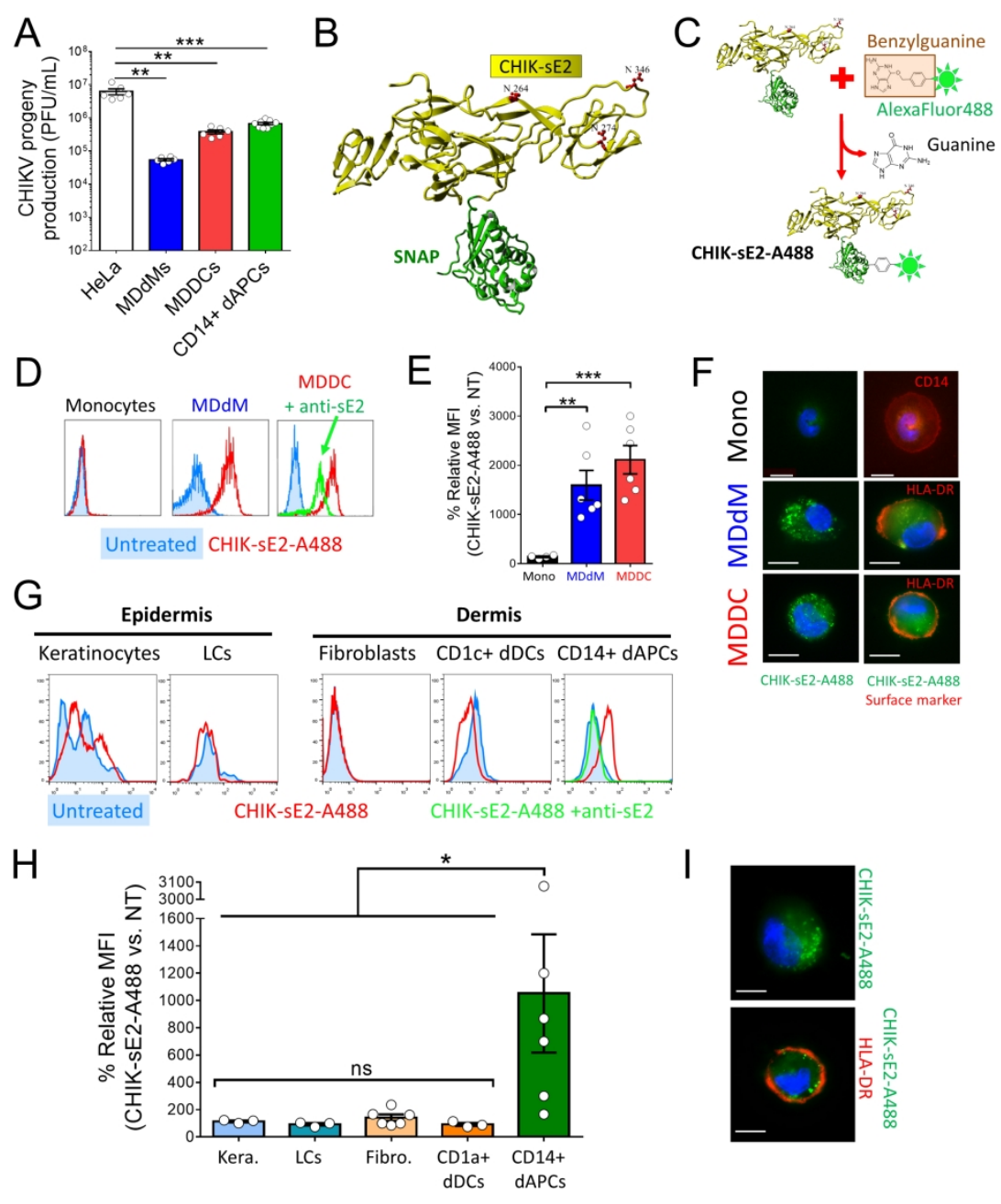

Figure 1

Figure 1: CD14+ dAPCs are infected by Chikungunya virus and selectively capture CHIK-sE2 recombinant envelope protein

(A) HeLa cells, MDdMs, MDDCs and CD14+ dAPCs were exposed to CHIKV at an MOI of 2.5. After 2h, the cells were washed and cultured for $24 \mathrm{~h}$. The cell supernatant was harvested and viral progeny titrated on AP-61 cells by a plaque assay. (B) Schematic representation of CHIK-sE2 cloned in frame with a SNAP-tag and produced in S2 insect cells. Red residues depict N-glycosylation sites N264, N274 and N346. (C) Covalent coupling to CHIK-sE2 occurs when the SNAP enzymatic moiety reacts with benzylguanine (BG)coupled AlexaFluor488, generating fluorescent CHIK-sE2-A488. (D) Interaction of CHIK-sE2-A488 with

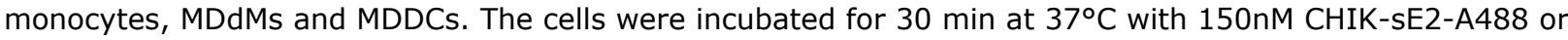
left untreated, then analyzed by flow cytometry. Representative histograms are shown. For MDDCs, CHIKsE2 was preincubated with or without anti-CHIK-sE2 mAb. (E) Relative mean fluorescence intensity (MFI) of CHIK-sE2-A488-treated cells vs. untreated cells ( $n=4-6$ donors). ( $F$ ) Confocal microscopy images of monocytes, MDdMs and MDDCs incubated with CHIK-sE2-A488. Counterstaining of CD14 or HLA-DR allowed visualization of the cell surface. DAPI labels nuclei in blue. Scale bars: $10 \mu \mathrm{m}$. (G) Healthy human skin 
samples were from donors undergoing plastic surgery. Epidermal cell suspensions were prepared by trypsin digestion. HLA-DR+ CD1a+ LCs and HLA-DR- CD1a- keratinocytes were identified by flow cytometry. The dermis was digested by collagenase and CD1c+ dDCs were isolated by anti-CD1c-coupled magnetic beads. After 2 days of culture, the dermal fibroblasts were identified as HLA-DR- CD14- adherent cells and the nonadherent CD14+ dAPCs were isolated by anti-CD14-coupled magnetic beads. 105 enriched cells were incubated in serum-free medium for $1 \mathrm{~h}$ at $37^{\circ} \mathrm{C}$ with $150 \mathrm{nM} \mathrm{CHIK}-\mathrm{sE} 2-\mathrm{A} 488$ or left untreated. Where indicated, CHIK-sE2-A488 was preincubated for 20min with anti-CHIK-sE2 mAb before addition of CD14+ dAPCs. Cells were stained with antibodies against HLA-DR, CD1a, CD14 and CD1C and analyzed by flow cytometry. $(\mathrm{H})$ Relative MFI comparing the fluorescence intensity of CHIK-sE2-A488-treated vs. untreated cells ( $n=3-6$ donors). (I) Confocal microscopy images of CD14+ dAPCs exposed to CHIK-sE2-A488 for 30 min at $37^{\circ} \mathrm{C}$. Cells were labelled for cell-surface HLA-DR, fixed and visualized by fluorescence microscopy. DAPI labels nuclei in blue. Scale bars: $10 \mu \mathrm{m}$. Data were obtained from at least three different donors and are presented as mean \pm SEM. Statistical comparisons: ANOVA followed by post-hoc tests (ns: non significant, $* p<0.05, * * p<0.01, * * * p<0.001)$. 

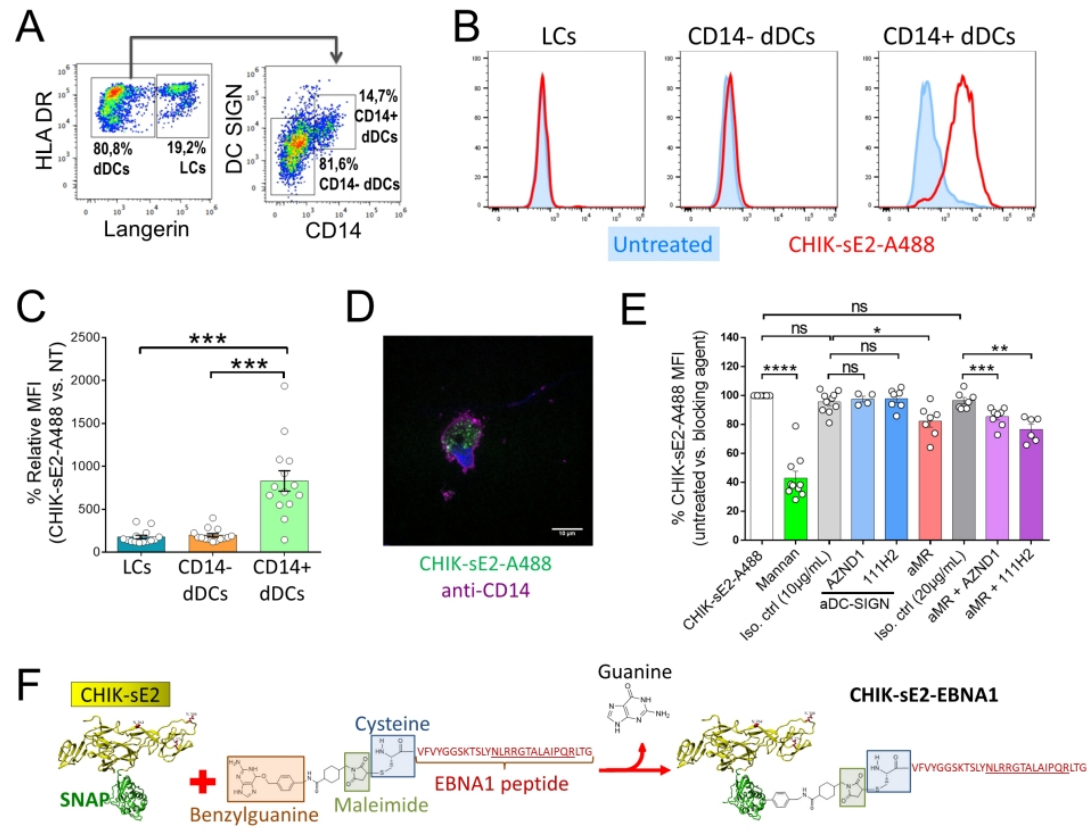
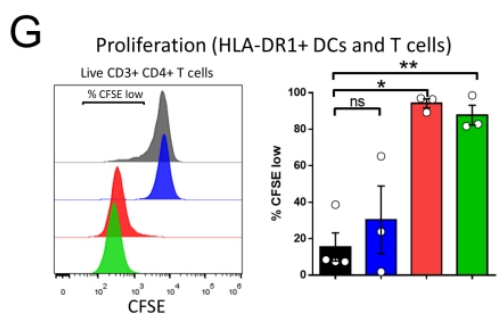

I

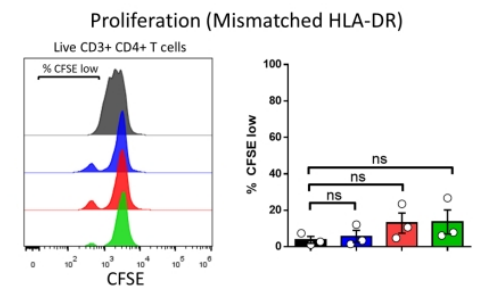

$\mathrm{H}$
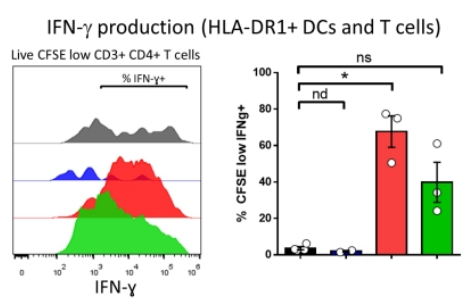

T cells alone

T cells + total skin DCs

T cells + total skin DCs loaded with CHIK-sE2-EBNA1

T cells + CD14+ dDCs loaded with CHIK-sE2-EBNA1

Figure 2

Figure 2: CHIK-sE2 conveys antigenic peptide to CD14+ dDCs for presentation to CD4+ T cells. (A) Whole skin pieces were floated on complete culture medium for 3 days. 105 total migratory cells were incubated alone or with $150 \mathrm{nM}$ CHIK-sE2-A488 in serum-free medium for $1 \mathrm{~h}$ at $37^{\circ} \mathrm{C}$. Cells were then stained with antibodies against HLA-DR, Langerin/CD207, DC-SIGN/CD209, and CD14 and analyzed by flow cytometry.

(B) Representative histograms of cells treated with CHIK-sE2-A488 or left untreated. (C) Relative MFI comparing the fluorescence intensity of CHIK-sE2-A488 vs. untreated cells ( $n=14$ donors). (D) CD14+ dDCs enriched by CD14 MACS were incubated with CHIK-sE2-A488 for $1 \mathrm{~h}$ at $37^{\circ} \mathrm{C}$, fixed with $4 \%$ PFA and stained for CD14. Fluorescent CHIK-sE2 in intracellular compartments was visualized by spinning-disk confocal microscopy. A video rendition is provided as Figure S3. (E) Skin migratory DCs ( $n=4-10$ donors) were incubated for $30 \mathrm{~min}$ at $37^{\circ} \mathrm{C}$ with $1 \mathrm{mM}$ mannan, $10 \mu \mathrm{g} / \mathrm{mL}$ purified antibodies against DC-SIGN (clones AZN-

D1 or $111 \mathrm{H} 2.02$ ), MR (clone 19.2), combinations of anti-MR and anti-DC-SIGN (total concentration $20 \mu \mathrm{g} / \mathrm{mL}$ ) or the corresponding isotype controls. CHIK-sE2-A488 binding was quantified as described above and expressed as \% of MFI obtained for untreated cells. (F) Schematic representation of EBNA1-derived peptide coupling to CHIK-sE2. The selected peptide contains the cognate HLA-DR1-restricted CD4+ T cell 
epitope (underlined). (G) EBV-specific T-cell proliferation induced by HLA-DR1-matched skin APCs with and without CHIK-sE2-EBNA1 loading (200nM) was measured by CFSE dilution assays. (H) IFN- $\square$ production was assessed by intracellular cytokine staining in proliferating EBNA1-specific CD4+ T cells after cognate peptide restimulation of co-cultures with the HLA-DR1-matched skin APCs with and without CHIK-sE2EBNA1 loading. (I) EBV-specific T-cell proliferation by HLA-DR-mismatched skin APCs was measured as in (G). Representative flow cytometry plots are shown for G, H, I. Data were obtained from at least three different donors and are presented as mean \pm SEM. Statistical comparisons: ANOVA followed by post-hoc tests (nd, not determined, ns: non significant, $* p<0.05, * * p<0.01, * * * p<0.001$ ).

$629 \times 900 \mathrm{~mm}(96 \times 96 \mathrm{DPI})$ 


\section{SUPPLEMENTARY FIGURE LEGENDS}

Figure S1: DC-SIGN and MR are receptors for CHIK-sE2. Affinity of CHIK-sE2 for immobilized MR (A) and DC-SIGN (B) were measured by surface plasmon resonance. Sensorgrams depict association and

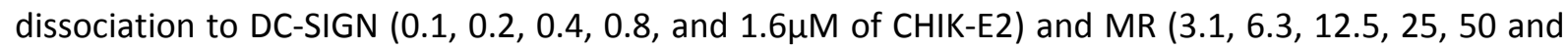
100nM of CHIK-sE2). (C) Constants for the binding assays. The KD for DC-SIGN and MR were 51.1nM and $3.9 \mathrm{nM}$, respectively.

Figure S2: CHIK-sE2 does not promote DC maturation. Immature MDDCs were incubated for $24 \mathrm{~h}$ with $150 \mathrm{nM}$ CHIK-sE2 or $100 \mathrm{ng} / \mathrm{mL}$ LPS. Maturation was quantified by flow cytometry from the MFI of CD86 stainings. Representative histograms are shown. Data were obtained from six different donors and are presented as mean $\pm S E M$. Statistical comparisons: Wilcoxon tests (ns: non significant, ${ }^{*} p<0.05$ ).

Figure S3: Z-stack video rendition of $\mathrm{CD}^{+} 4^{+} \mathrm{dDCs}$ loaded with CHIK-sE2-A488. CD14 ${ }^{+} \mathrm{dDC}$ enriched by CD14 MACS were incubated with 150nM CHIK-sE2-A488 in serum-free medium for $1 \mathrm{~h}$ at $37^{\circ} \mathrm{C}$, then fixed with 4\% PFA and counterstained with anti-CD14 to highlight the cell surface (magenta). Fluorescent CHIK-sE2 (green) was visualized in intracellular compartments of targeted cells by spinning-disk confocal microscopy.

\section{SUPPLEMENTARY MATERIAL AND METHODS}

\section{Antibodies and media}

The following antibodies were used for flow cytometry: HLA-DR (clones Tü39 or L243), CD206/MR (19.2), CD209/DC-SIGN (DCN46), CD1a (HI149), CD86 (FUN-1), IFN-ץ (B27), CD3 (UCHT1) from BD, CD14 (MEM-18, Immunotools), CD1C (AD5-8E7) and CD4 (REA623) from Miltenyi Biotec, and CD207/Langerin (DCGM4, Dendritics). Staining buffer was PBS containing $2 \%$ fetal calf serum (FCS; Dutscher) and 2.5mM EDTA (SE buffer). Dead cells were labelled by Fixable Viability Dye-eFluor450 (eBioscience) or Sytox Red dye (Invitrogen). Cells were collected on FACSCalibur (BD) or Gallios (Beckman-Coulter) flow cytometers. Data were analysed with CellQuest (BD), Kaluza (BeckmanCoulter) or FlowJo (Treestar) softwares. Complete medium was RPMI1640 supplemented with $10 \mu \mathrm{g} / \mathrm{mL}$ gentamycin, 100 units $/ \mathrm{mL}$ penicillin, $100 \mu \mathrm{g} / \mathrm{mL}$ streptomycin, $10 \mathrm{mM}$ HEPES (all from Lonza) and $10 \%$ FCS.

\section{Primary cell isolation and culture}

Fresh abdominal skin was obtained from patients undergoing abdominoplasty with written informed consent and institutional review board approval, in agreement with the Helsinki Declaration and French legislation. 
Enzymatic digestion: Subcutaneous fat was removed and the remainder was incubated for $1 \mathrm{~h}$ at $37^{\circ} \mathrm{C}$ in PBS containing 0.5\% trypsin XI (Sigma-Aldrich). The epidermis was then peeled off and agitated in complete medium for $10 \mathrm{~min}$ to liberate LCs and keratinocytes. The dermis was cut into small pieces and digested overnight with $1 \mathrm{mg} / \mathrm{mL}$ collagenase I (Invitrogen) and $50 \mu \mathrm{g} / \mathrm{mL}$ DNAse (Sigma-Aldrich) at $37^{\circ} \mathrm{C}$ and $5 \% \mathrm{CO}_{2}$ in complete medium. The digested dermis was filtered in SE buffer with $50 \mu \mathrm{m}$ cell strainers and dermal CDC2 were isolated by anti-CD1c-coupled magnetic beads (Miltenyi Biotec). Remaining cells were incubated for $48 \mathrm{~h}$ in a $175 \mathrm{~cm}^{2}$ culture flask $\left(50 \times 10^{6}\right.$ cells $/ 50 \mathrm{~mL}$ complete medium). CD14 ${ }^{+}$dAPCs were isolated from the loosely adherent cells using anti-CD14-conjugated magnetic beads (Miltenyi Biotec). After an overnight culture in dermal fibroblast-conditioned culture medium at $10^{6}$ cells $/ \mathrm{mL}, \mathrm{CD} 14^{+} \mathrm{dAPC}$ were sorted by flow cytometry as CD14 ${ }^{+} \mathrm{HLA}-\mathrm{DR} \mathrm{R}^{\text {low }} \mathrm{CD} 86^{\text {low }}$ cells. Skin explant cultures: $2 \mathrm{~cm}^{2}$ biopsies of $\sim 1 \mathrm{~mm}$ thickness were placed at air-liquid interface onto $40 \mu \mathrm{m}$ cell strainers in $6 \mathrm{~mL}$ complete medium. After 3 days, migratory cells were harvested from the culture supernatant. Resident memory $\mathrm{T}$ cells were excluded by selection with anti-CD3 magnetic beads (Miltenyi-Biotec). Where indicated, $\mathrm{CD} 14^{+} \mathrm{dDCs}$ were separated with anti-CD14 magnetic beads (Miltenyi-Biotec).

\section{Monocyte-derived DCs and macrophages}

Monocyte-derived dermal-like macrophages (MDdMs) and MDDCs were generated from blood monocytes as described (Kwan et al., 2008). Briefly, $2 \times 10^{6}$ monocytes, purified from adult blood by counterflow centrifugal elutriation (Etablissement Français du Sang, Strasbourg, France) were cultured in $5 \mathrm{~mL}$ complete medium in the presence of GM-CSF $(50 \mathrm{ng} / \mathrm{mL})$ and IL-4 $(10 \mathrm{ng} / \mathrm{mL})$ for MDDCs, or GMCSF $(25 \mathrm{ng} / \mathrm{mL})$, IL-10 $(25 \mathrm{ng} / \mathrm{mL})$ and M-CSF $(10 \mathrm{ng} / \mathrm{mL})$ for MDdMs. On day 3, the culture was refreshed with $1 \mathrm{~mL}$ of complete medium containing GM-CSF $(50 \mathrm{ng} / \mathrm{mL})$ and IL-4 $(10 \mathrm{ng} / \mathrm{mL})$ for MDDCs, or GMCSF $(12.5 \mathrm{ng} / \mathrm{mL})$ and IL-10 $(12.5 \mathrm{ng} / \mathrm{mL})$ for MDdMs. On day 5 , cells were characterized by flow cytometry for expression of CD1a, CD14 and DC-SIGN.

\section{Cell lines}

HeLa and Vero cells were cultured at $37^{\circ} \mathrm{C}$ and $5 \% \mathrm{CO}_{2}$ in DMEM (Invitrogen) supplemented with $10 \%$ FCS and antibiotics. Aedes pseudoscutellaris AP-61 mosquito cells were cultured at $27^{\circ} \mathrm{C}$ in Leibovitz's L-15 medium (Invitrogen) supplemented with 10\% FCS, 1\% tryptose phosphate broth (Eurobio) and antibiotics.

\section{Viral infections}

The CHIKV-06.049 virus strain was isolated from a young adult during the 2006 outbreak of Chikungunya fever in La Réunion island (Schuffenecker et al., 2006). Virus stocks were produced on mosquito AP-61 cells (Brehin et al., 2008) and all infectivity titers were expressed as plaque-forming units on Vero cells. $5 \times 10^{4}$ HeLa cells, CD14 ${ }^{+}$dAPCs, MDdMs and MDDC were exposed to CHIKV at an $\mathrm{MOI}$ of 2.5 , or mock-infected, in RPMI 1640 supplemented with $2 \% \mathrm{FCS}$ for $2 \mathrm{~h}$ at $37^{\circ} \mathrm{C}$. Virus was 
removed by extensive washing and cells were kept for $22 \mathrm{~h}$ in their respective culture media. The supernatants were used for titer analysis by the plaque-forming assay on Vero cells.

\section{Recombinant CHIK-sE2 protein production}

Stably transfected Drosophila melanogaster S2 cell line inducible for the secretion of the ectodomain of CHIKV envelope E2 protein (CHIK-sE2; residues 4-364) were described elsewhere (Aubry et al., 2015). Briefly, a SNAP adaptor sequence (New England Biolabs) was fused in-frame with the C-terminus of recombinant CHIK-sE2, resulting in a chimeric protein that was purified from the supernatants of S2 cells. SNAP is the catalytic site of O6-alkylguanine-DNA-alkyltransférase. 3D rendition of CHIK-sE2SNAP was created with the Yasara software based on the available crystal structures (CHIK-sE2: PDB 3J2W; SNAP-tag: PDB 3KZY).

\section{Surface plasmon resonance}

Surface plasmon resonance experiments were performed using a Biacore 3000. Extracellular domains of DC-SIGN[Lys62-Ala404] and MR[Leu19-Lys1383] (R\&D Systems) were immobilized on a CM5 sensor chip (GE-Healthcare) by injecting $70 \mu \mathrm{L}$ of recombinant proteins $(50 \mu \mathrm{g} / \mathrm{mL}$ in formate buffer, $\mathrm{pH} 4.3$ ) onto the surface activated with $\mathrm{N}$-Ethyl-N'-dimethylaminopropyl carbodiimide/N-hydroxysuccinimide. $20 \mu \mathrm{L}$ of ethanolamine hydrochloride, $\mathrm{pH} 8.5$, saturated the free activated sites of the matrix. Biosensor assays were performed with HEPES-buffered-saline (10mM HEPES, 150mM sodium chloride, pH 7.4) containing $0.005 \%$ surfactant $\mathrm{P} 20$ and $5 \mathrm{mM} \mathrm{CaCl} 2$, as running buffer. All binding experiments were carried out at $25^{\circ} \mathrm{C}$ with a constant flow rate of $20 \mu \mathrm{L} / \mathrm{min}$. CHIK-sE2 dissolved in the running buffer was injected for $3 \mathrm{~min}$, followed by a dissociation phase of $3 \mathrm{~min}$. The sensor chip surface was regenerated after each experiment by injecting $10 \mu \mathrm{L}$ EDTA $0.5 \mathrm{M} \mathrm{pH}$ 8.0. The kinetic parameters were calculated using the BIAeval 4.1 software. Analysis was performed using the simple Langmuir binding 1:1 model with separate $\mathrm{ka} / \mathrm{kd}$ (kon/koff). The specific binding profiles were obtained after subtracting the response signal from the control channel and from blank-buffer injection. The fitting to each model was judged by the chi-square value and randomness of residue distribution compared to the theoretical model.

\section{CHIK-sE2 binding studies}

Benzylguanine (BG)-AlexaFluor488 fluorochrome (New England Biolabs) was covalently linked to CHIKSE2 via the SNAP moiety according to the manufacturer's recommendations. $10^{5}$ cells were incubated in $200 \mu \mathrm{L}$ RPMI1640 alone or with $2 \mu \mathrm{g}$ of CHIK-sE2-A488 (final concentration $150 \mathrm{nM}$ ) for $30 \mathrm{~min}$ at $37^{\circ} \mathrm{C}$. The cells were then washed and labelled for cell surface markers in SE buffer and analyzed by flow cytometry. Where indicated, CHIK-sE2 $(1.2 \mu \mathrm{g})$ was pre-incubated for $30 \mathrm{~min}$ at $4^{\circ} \mathrm{C}$ with anti-E2

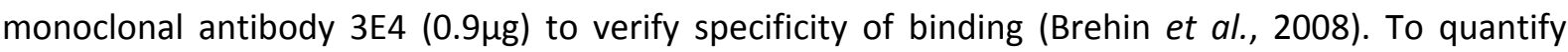
binding efficiency, the mean fluorescent intensity (MFI) of CHIK-sE2-A488-exposed cells was calculated relatively to the MFI of untreated cells, which was set at $100 \%$. For internalization studies by confocal 
microscopy, cells exposed to CHIK-sE2-A488 were washed in SE buffer and counterstained with antibodies against HLA-DR or CD14 for $25 \mathrm{~min}$ on ice. The cells were washed twice, fixed in $4 \%$ formaldehyde for $20 \mathrm{~min}$ at RT, and the secondary goat-anti-mouse AlexaFluor594 antibody (Invitrogen) was added and incubated for 30min on ice. After washing, nuclei were labelled with DAPI. Samples were mounted on glass slides in fluorescence mounting medium (Dako). Images taken with Zeiss confocal microscopes LSM510 and Axio Observer Z1 were analyzed with ImageJ. For maturation assays, MDDCs were incubated with 150nM CHIK-sE2 or 100ng/mL ultrapure K12 LPS (Invivogen) for $24 \mathrm{~h}$, then CD86 expression was quantified by flow cytometry.

\section{Peptide synthesis}

Peptides were assembled using optimized Fmoc chemistry protocols with a multichannel peptide synthesizer (Neimark and Briand, 1993). Side-chain deprotection and cleavage of peptides from the solid support was performed by treatment with reagent $\mathrm{K}$ (82.5\% TFA v/v, 5\% phenol w/v, 5\% water $\mathrm{v} / \mathrm{v}, 5 \%$ thioanisole $\mathrm{v} / \mathrm{v}, 2.5 \%$ 1,2-ethanedithiol $\mathrm{v} / \mathrm{v}$ ) for $150 \mathrm{~min}$ at $20^{\circ} \mathrm{C}$. Peptides were purified by reversed-phase HPLC (RP-HPLC) using a Beckman preparative HPLC system (Gagny, France) on a Nucleosil C18 $(1 \times 30 \mathrm{~cm})$ column, (Macherey Nagel, Hoerdt, France). The elution was achieved with a linear gradient of aqueous $0.1 \%$ TFA and $0.08 \%$ TFA in acetonitrile at a flow rate of $6 \mathrm{~mL} / \mathrm{min}$ with UV detection at $230 \mathrm{~nm}$. The purity of peptides was controlled by analytical RP-HPLC on a Beckman instrument (Gagny, France) with a Nucleosil C18 $5 \mu \mathrm{m}$ column $(150 \times 4.6 \mathrm{~mm})$ using a linear gradient of $0.1 \%$ TFA in water and acetonitrile containing $0.08 \%$ TFA at a flow rate of $1.2 \mathrm{~mL} / \mathrm{min}$. The integrity of each peptide was assessed by LC/MS using a Thermo Finigan LCQ.

The Epstein-Barr virus Nuclear Antigen-1 (EBNA1)-derived peptide (507-535; CVFVYGGSKTSLYNLRRGTALAIPQRLTG) was bound to BG-maleimide (New England Biolabs) by reacting with the thiol group of the N-terminal cysteine residue, before purification by HPLC.

\section{Blocking CHIK binding}

Skin migratory DCs were incubated for $30 \mathrm{~min}$ at $37^{\circ} \mathrm{C}$ with $1 \mathrm{mM}$ mannan (Sigma-Aldrich), $10 \mu \mathrm{g} / \mathrm{mL}$ purified antibodies against DC-SIGN (clone AZN-D1, Dr. Teunis Geijtenbeek, Amsterdam, NL (Geijtenbeek et al., 2000); clone 111H2.02, Dendritics, France (Canard et al., 2011)), MR (clone 19.2, $\mathrm{BD}$ ), combinations of anti-MR and anti-DC-SIGN (total concentration $20 \mu \mathrm{g} / \mathrm{mL}$ ) or the corresponding isotype controls. Then, $150 \mathrm{nM}$ CHIK-sE2-A488 was added for $1 \mathrm{~h}$ and its binding was quantified by flow cytometry.

\section{Expansion of EBNA1-specific CD4+ T memory cells}

Allogeneic PBMCs were purified from buffy coats of healthy blood donors (EFS) by Ficoll gradient (Sigma-Aldrich). $5 \times 10^{3}$ EBNA1-specific CD4 ${ }^{+}$T cell (CK-NLR clone, HLA-DR1-restricted) cells/well were cocultured with $10^{5}$ irradiated PBMCs (3000rad) and $2 \times 10^{3}$ irradiated, EBV-infected autologous B-LCL (20000rad) in RPMI1640 supplemented with 8\% PHS (pooled human sera, Corning), 150U/mL IL-2 
(Novartis), $1 \mu \mathrm{g} / \mathrm{mL}$ Phytohemagglutinin-L (Sigma-Aldrich), and gentamicin. After 5 days, half of the medium was refreshed with RPMI $1640+8 \%$ PHS + gentamycin $+450 \mathrm{U} / \mathrm{mL}$ IL-2. Cells were monitored daily and split into the same medium and new 96 well-plates if necessary, until freezing at day 14 (Rühl et al., 2019).

\section{Antigen presentation assays}

Genomic DNA was extracted from 40-80mg of skin using Nucleospin columns (Macherey-Nagel) and HLA haplotype of donors was determined with SSP HLA DQDR Combitray kit (Olerup). BG-EBNA1 peptide (250pmol) was coupled to CHIK-sE2 (200pmol) via the SNAP moiety in PBS. CHIK-sE2-EBNA1 (200nM) was incubated with $5 \times 10^{5}$ migratory skin DCs or CD14 ${ }^{+}$dDCs for $1 \mathrm{~h}$ in RPMI1640 medium. $10^{4}$ T-cell depleted, CHIK-EBNA1-loaded skin DCs or CD14+ dDCs from HLA-DR1 donors were cocultured in $200 \mu \mathrm{L}$ RPMI1640 + 8\% PHS in 96-well U-bottom plates with $5 \times 10^{4}$ EBNA1-specific $\mathrm{CD}^{+}{ }^{+}$cells previously labelled with $0.5 \mu \mathrm{M}$ carboxyfluorescein succinimidyl ester (CFSE). After 5 days, T cells were restimulated $6 \mathrm{~h}$ with EBNA1 peptide $(1 \mu \mathrm{g} / \mathrm{mL})$, including $4 \mathrm{~h}$ with $10 \mu \mathrm{g} / \mathrm{mL}$ brefeldin $\mathrm{A}$ and $1 \mu \mathrm{M}$ monensin (BD). $T$ cells were stained for $C D 3$ and $C D 4$, then fixed and permeabilized with $B D$ Cytofix/Cytoperm Kit, stained with anti-IFN- $\nu$ antibody and analyzed by flow cytometry. T-cell proliferation was determined by CFSE dilution.

\section{Statistical analyses}

Data from different experimental conditions were tested by ANOVA, followed by relevant post-hoc multiple comparisons or by Wilcoxon tests, performed with Prism 6.0 software (GraphPad). Values of p below 0.05 were considered significant. 


\section{REFERENCES}

Aubry M, Finke J, Teissier A, Roche C, Broult J, Paulous S, et al. Silent Circulation of Ross River Virus in French Polynesia. Int J Infect Dis 2015;37:19-24.

Brehin AC, Rubrecht L, Navarro-Sanchez ME, Marechal V, Frenkiel MP, Lapalud P, et al. Production and characterization of mouse monoclonal antibodies reactive to Chikungunya envelope E2 glycoprotein. Virology 2008;371:185-195.

Canard B, Vachon H, Fontaine T, Pin JJ, Paul S, Genin C, et al. Generation of anti-DC-SIGN monoclonal antibodies capable of blocking HIV-1 gp120 binding and reactive on formalin-fixed tissue. Immunol Lett 2011;135:165-172.

Geijtenbeek TBH, Torensma R, Van Vliet SJ, Van Duijnhoven GCF, Adema GJ, Van Kooyk Y, et al. Identification of DC-SIGN, a novel dendritic cell-specific ICAM-3 receptor that supports primary immune responses. Cell 2000;100:575-585.

Kwan WH, Navarro-Sanchez E, Dumortier H, Decossas M, Vachon H, dos Santos FB, et al. Dermal-type macrophages expressing CD209/DC-SIGN show inherent resistance to dengue virus growth. PLoS NegI Trop Dis 2008;2:e311.

Neimark J, Briand JP. Development of a fully automated multichannel peptide synthesizer with integrated TFA cleavage capability. Pept Res 1993;6:219-228.

Rühl J, Citterio C, Engelmann C, Haigh T, Dzionek A, Dreyer J, et al. Heterologous prime-boost vaccination protects against EBV antigen-expressing lymphomas. J Clin Invest 2019;129:2071-2087.

Schuffenecker I, Iteman I, Michault A, Murri S, Frangeul L, Vaney MC, et al. Genome microevolution of chikungunya viruses causing the Indian Ocean outbreak. PLoS Med 2006;3:e263. 

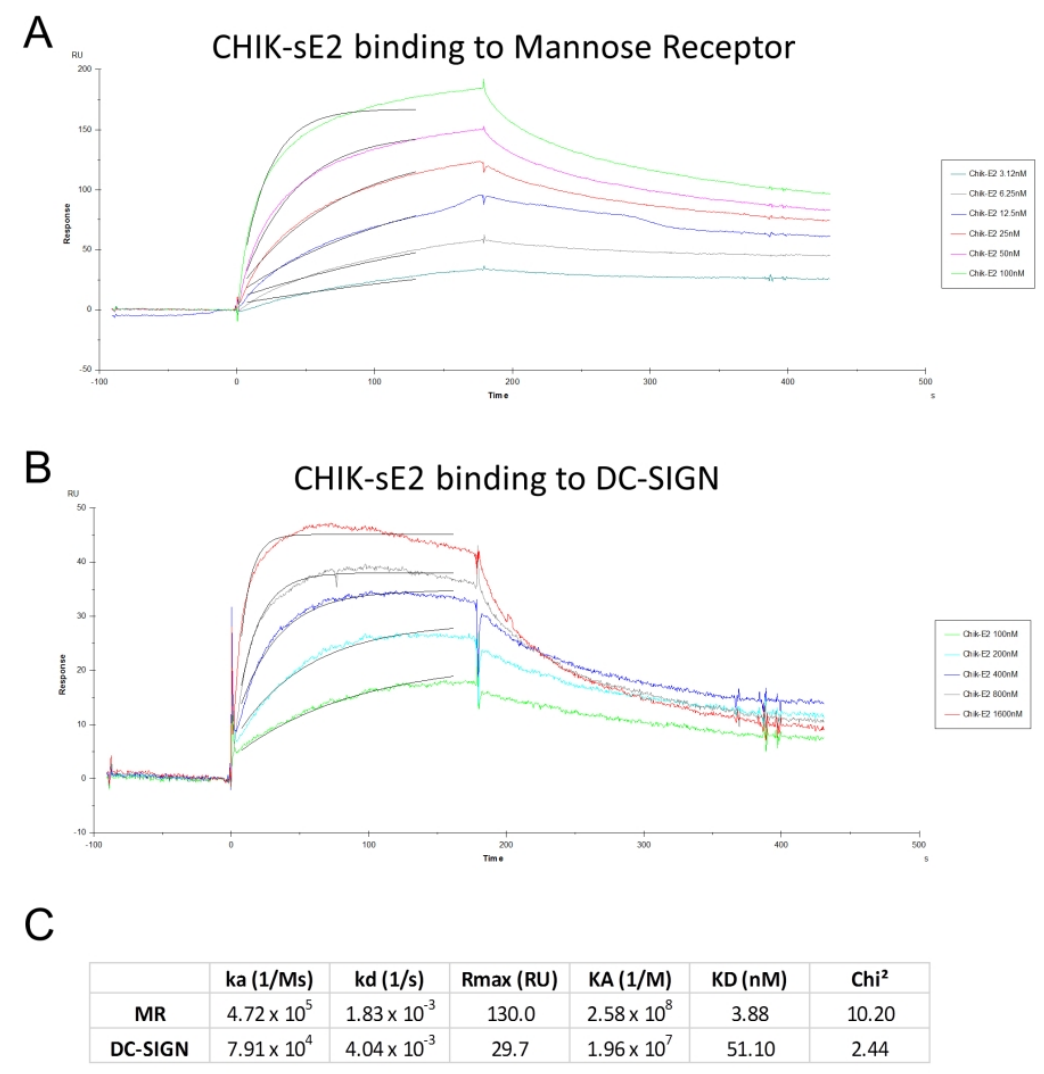

\section{Supplementary Figure S1}

Figure S1: DC-SIGN and MR are receptors for CHIK-sE2. Affinity of CHIK-sE2 for immobilized MR (A) and DC-SIGN (B) were measured by surface plasmon resonance. Sensorgrams depict association and dissociation to DC-SIGN $(0.1,0.2,0.4,0.8$, and $1.6 \mu \mathrm{M}$ of CHIK-E2) and MR $(3.1,6.3,12.5,25,50$ and $100 \mathrm{nM}$ of CHIK-sE2). (C) Constants for the binding assays. The KD for DC-SIGN and MR were $51.1 \mathrm{nM}$ and $3.9 \mathrm{nM}$, respectively. 

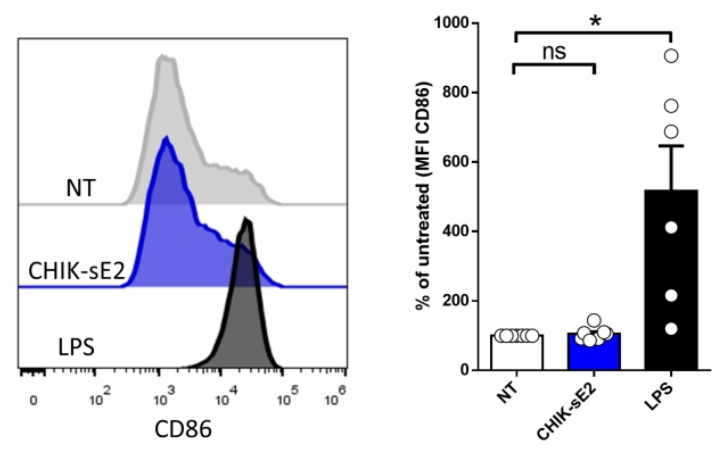

Supplementary Figure S2

Figure S2: CHIK-sE2 does not promote DC maturation. Immature MDDCs were incubated for $24 \mathrm{~h}$ with $150 \mathrm{nM}$ CHIK-sE2 or $100 \mathrm{ng} / \mathrm{mL}$ LPS. Maturation was quantified by flow cytometry from the MFI of CD86 stainings. Representative histograms are shown. Data were obtained from six different donors and are presented as mean \pm SEM. Statistical comparisons: Wilcoxon tests (ns: non significant, $* p<0.05$ ). 